\title{
Delay-dependent non-fragile robust dissipative filtering for uncertain nonlinear stochastic singular time-delay systems with Markovian jump parameters
}

Yuechao Ma and Huijie Yan*

"Correspondence:

18716023220@163.com College of Science, Yanshan University, 438 Hebeidajie Road, Haigang District, Qinhuangdao, Hebei 066004, P.R. China

\begin{abstract}
The problem of delay-dependent non-fragile and robust dissipative filtering is investigated for a class of uncertain nonlinear stochastic singular time-delay Itô-type systems with Markovian jump parameters. With the system uncertainty and the filter gain perturbations, the closed-loop filtering error system is robust asymptotically stable and satisfies the dissipation performance. By constructing a Lyapunov-Krasovskii function and applying the Itô differential formula to compute the differential function along the system, a sufficient condition for the existence of a robust and non-fragile dissipative filter is derived in the form of linear matrix inequality (LMI). An illustrative numerical example is provided to demonstrate the effectiveness of the proposed approach.
\end{abstract}

Keywords: uncertain nonlinear singular time-delay system; stochastic system; Markov switching; non-fragile robust dissipative filtering

\section{Introduction}

As an important kind of hybrid systems, Markovian jump systems have received increasing attention in the past few years due to the fact that they have strong practical relevance in mechanical systems, economics systems with human operators, and other engineering areas [1-4]. Partially mode-dependent $H_{\infty}$ filter design problem was tackled for discretetime Markovian jump systems with partly unknown transition probabilities in [5].

Note that stochastic systems with Brownian motions, governed by the Itô differential equations, have attracted many researchers' attention over the past decades due to the extensive application of stochastic modeling in mechanical systems, economics, and other areas. In [6], the $H_{\infty}$ filtering problem was solved for uncertain stochastic time-delay systems with sector-bounded nonlinearities. In [7], the joint state filtering and parameter estimation problem was solved for linear stochastic time-delay systems. In [8], the robust $H_{\infty}$ filtering problem was solved for discrete nonlinear stochastic systems with time-varying delay. In [9], the problem of non-fragile $H_{\infty}$ filtering was thoroughly studied for uncertain stochastic time-delay systems.

For the stochastic case, the Markovian switching problem has received considerable attention, and a number of traditional approaches have been proposed in the literature. In

() 2013 Ma and Yan; licensee Springer. This is an Open Access article distributed under the terms of the Creative Commons Attribution License (http://creativecommons.org/licenses/by/2.0), which permits unrestricted use, distribution, and reproduction in any medium, provided the original work is properly cited. 
[10], the delay-range-dependent robust $H_{\infty}$ filtering problem was neatly solved for uncertain stochastic systems with mode-dependent time delays and Markovian jump parameters. In [11], the non-fragile $H_{\infty}$ filtering problem for uncertain stochastic time-delay systems with Markovian jump parameters was thoroughly studied. In [12], the robust $H_{\infty}$ exponential filtering was exploited to uncertain stochastic time-delay systems with Markovian switching and nonlinearities. It is well known that stochastic time-delay systems with Markovian jump parameters play a very important role in digital signal analysis and processing. However, despite its importance, up to now, the delay-dependent non-fragile robust dissipative filtering problem for general uncertain stochastic time-delay systems with Markovian jump parameters has not been fully investigated and the relevant results have been very few.

On the other hand, a great deal of attention has been devoted to the study of singular systems over the past decades. Singular systems are referred to as descriptor systems, implicit systems, generalized state-space systems, or semi-state systems. The singular Markovian jump time-delay systems have also been investigated by many researchers. In [13], the robust exponential stability was studied for uncertain singular Markovian jump time-delay systems. In [14], the delay-dependent $H_{\infty}$ filtering problem for singular Markovian jump time-delay systems was thoroughly investigated.

The notion of dissipativity plays an important role in systems, circuits, networks, and control theory. The dissipativity contains small gain and passivity as its special cases and is mainly used for stability analysis for nonlinear systems. The main motivation for the study of a general dissipative control problem is that it offers flexibility for gain and phase performance trade off. Moreover, it is effective to deal with robust and nonlinear control. In [15], the passivity control for a kind of T-S fuzzy descriptor system is presented. In [16], the dissipative filtering for discrete fuzzy systems was thoroughly studied. In [17], the dissipative filtering for linear discrete-time systems via LMI was studied. In [18], the robust dissipative filtering for continuous-time polytopic uncertain neutral systems was studied. Unfortunately, to the best of the authors' knowledge, up to now, the delay-dependent nonfragile robust dissipative filtering for uncertain nonlinear stochastic singular time-delay systems with Markovian jump parameters has rarely been reported.

In this paper, the delay-dependent non-fragile robust dissipative filter design method for uncertain nonlinear stochastic singular time-delay systems with Markovian jump parameters is considered. The system under study involves parameter uncertainties, stochastic disturbances, time-varying delays and inherent sector-like nonlinearities. Note that, among different descriptions of the nonlinearities, the so-called sector nonlinearity [19] has gained much attention for deterministic systems, the control analysis and model reduction problems have been studied; see [20, 21]. By establishing a Lyapunov-Krasovskii function and applying the Itô differential formula, a new delay-dependent bounded real lemma for nonlinear stochastic singular time-delay and Markovian jump systems is derived. Neither model transformation nor bounding technique for cross terms is involved. Based on the obtained bounded real lemma, the existence condition of a robust dissipative filter and a filter design method are presented by LMI approach. Also, it is shown that the proposed filter design method is widely applicable to singular systems and non-singular systems by a numerical example. 


\section{System description and preliminary lemma}

Given a probability space $(\Omega, F, P)$, where $\Omega$ is the sample space, $F$ is the $\sigma$ algebra of subsets of the sample space and $P$ is the probability measure on $F$. Over this probability space $(\Omega, F, P)$, we consider the following uncertain linear stochastic systems with Markovian jump parameters and mode-dependent time delays:

$$
\begin{aligned}
& E d x(t)= {\left[A(r(t), t) x(t)+A_{d}(r(t), t) x(t-d(t))+B(r(t), t) f(x(t))\right.} \\
&\left.+B_{d}(r(t), t) f_{d}(x(t-d(t)))+C(r(t), t) v(t)\right] d t \\
&+\left[G(r(t), t) x(t)+G_{d}(r(t), t) x(t-d(t))+H(r(t), t) f(x(t))\right. \\
&\left.+H_{d}(r(t), t) f_{d}(x(t-d(t)))+D(r(t), t) v(t)\right] d \omega(t), \\
& d y(t)=[ \bar{A}(r(t), t) x(t)+\bar{A}_{d}(r(t), t) x(t-d(t))+\bar{B}(r(t), t) f(x(t)) \\
&\left.+\bar{B}_{d}(r(t), t) f_{d}(x(t-d(t)))+\bar{C}(r(t), t) v(t)\right] d t \\
&+\left[\bar{G}(r(t), t) x(t)+\bar{G}_{d}(r(t), t) x(t-d(t))+\bar{H}(r(t), t) f(x(t))\right. \\
&\left.+\bar{H}_{d}(r(t), t) f_{d}(x(t-d(t)))+\bar{D}(r(t), t) v(t)\right] d \omega(t), \\
& z(t)=L(r(t), t) x(t)+J(r(t), t) x(t-d(t))+B_{z}(r(t), t) f(x(t))+H_{z}(r(t), t) f_{d}(x(t-d(t)))+K(r(t), t) v(t), \\
& x(t)=\varphi(t), \quad t \in[-d, 0],
\end{aligned}
$$

where $x(t) \in R^{n}$ is the state; $v(t) \in R^{p}$ is the disturbance input which belongs to $L_{2}[0, \infty)$; $y(t) \in R^{p}$ is the measurement; $z(t) \in R^{q}$ is the signal to be estimated; and $\omega(t)$, independent of the Markov process, is a one-dimensional standard Wiener process. $E$ is a singular square matrix, and $\operatorname{rank} E=r<n . A(r(t), t), A_{d}(r(t), t), B(r(t), t), B_{d}(r(t), t)$, $C(r(t), t), G(r(t), t), G_{d}(r(t), t), H(r(t), t), H_{d}(r(t), t), D(r(t), t), \bar{A}(r(t), t), \bar{A}_{d}(r(t), t), \bar{B}(r(t), t)$, $\bar{B}_{d}(r(t), t), \bar{C}(r(t), t), \bar{G}(r(t), t), \bar{G}_{d}(r(t), t), \bar{H}(r(t), t), \bar{H}_{d}(r(t), t), \bar{D}(r(t), t), L(r(t), t), J(r(t), t)$, $B_{z}(r(t), t), H_{z}(r(t), t), K(r(t), t)$ are governed by the Markov process $r(t)$, and $K(r(t), t)$ is symmetric. $r(t)$ is a continuous-time Markovian process with right-continuous trajectories and taking values in a finite set $S=\{1,2, \ldots, N\}$ with transition probability matrix $\Pi=\left[\pi_{i j}\right]_{i, j \in S}$ given by

$$
P\{r(t+\Delta)=j \mid r(t)=i\}= \begin{cases}\pi_{i j} \Delta+o(\Delta), & i \neq j, \\ 1+\pi_{i j} \Delta+o(\Delta), & i=j,\end{cases}
$$

where $\Delta>0, \lim _{\Delta \rightarrow 0} \frac{o(\Delta)}{\Delta}=0 ; \pi_{i j}$ for $i \neq j$ is the transition rate from mode $i$ at time $t$ to mode $j$ and $\pi_{i i}=-\sum_{i \neq j} \pi_{i j} . d(t)$ is the time-varying delay when the mode is in $r(t)$ and satisfies

$$
0 \leq d_{i}(t) \leq d_{1 i}, \quad \dot{d}_{i}(t) \leq h_{i}<1, \quad \forall r(t)=i, i \in S,
$$

where $d_{1 i}, h_{i}$ are known real constant scalars, $d_{1}=\max \left\{d_{1 i}, i \in S\right\} . \varphi(t)$ denotes a vectorvalued initial continuous function defined on the interval $\left[-d_{1}, 0\right]$. 
When $r(t)=i$, let $A_{i}$ denote $A(r(t), t)$; so, for each $r(t)=i \in S$,

$$
\begin{array}{lcc}
A(r(t), t)=A_{i}+\Delta A_{i}, & A_{d}(r(t), t)=A_{d i}+\Delta A_{d i}, & B(r(t), t)=B_{i}+\Delta B_{i}, \\
B_{d}(r(t), t)=B_{d i}+\Delta B_{d i}, & C(r(t), t)=C_{i}+\Delta C_{i}, & G(r(t), t)=G_{i}+\Delta G_{i}, \\
G_{d}(r(t), t)=G_{d i}+\Delta G_{d i}, & H(r(t), t)=H_{i}+\Delta H_{i}, & H_{d}(r(t), t)=H_{d i}+\Delta H_{d i}, \\
D(r(t), t)=D_{i}+\Delta D_{i}, & \bar{A}(r(t), t)=\bar{A}_{i}+\Delta \bar{A}_{i}, & \bar{A}_{d}(r(t), t)=\bar{A}_{d i}+\Delta \bar{A}_{d i}, \\
\bar{B}(r(t), t)=\bar{B}_{i}+\Delta \bar{B}_{i}, & \bar{B}_{d}(r(t), t)=\bar{B}_{d i}+\Delta \bar{B}_{d i}, & \bar{C}(r(t), t)=\bar{C}_{i}+\Delta \bar{C}_{i}, \\
\bar{G}(r(t), t)=\bar{G}_{i}+\Delta \bar{G}_{i}, & \bar{G}_{d}(r(t), t)=\bar{G}_{d i}+\Delta \bar{G}_{d i}, & \bar{H}(r(t), t)=\bar{H}_{i}+\Delta \bar{H}_{i}, \\
\bar{H}_{d}(r(t), t)=\bar{H}_{d i}+\Delta \bar{H}_{d i}, & \bar{D}(r(t), t)=\bar{D}_{i}+\Delta \bar{D}_{i}, & L(r(t), t)=L_{i}+\Delta L_{i}, \\
J(r(t), t)=J_{i}+\Delta J_{i}, & B_{z}(r(t), t)=B_{z i}+\Delta B_{z i}, & H_{z}(r(t), t)=H_{z i}+\Delta H_{z i}, \\
K(r(t), t)=K_{i}+\Delta K_{i}, &
\end{array}
$$

where $A_{i}, A_{d i}, B_{i}, B_{d i}, C_{i}, G_{i}, G_{d i}, H_{i}, H_{d i}, D_{i}, \bar{A}_{i}, \bar{A}_{d i}, \bar{B}_{i}, \bar{B}_{d i}, \bar{C}_{i}, \bar{G}_{i}, \bar{G}_{d i}, \bar{H}_{i}, \bar{H}_{d i}, \bar{D}_{i}, L_{i}, J_{i}$, $B_{z i}, H_{z i}, K_{i}$ are known real constant matrices describing the nominal system; $\Delta A_{i}, \Delta A_{d i}$, $\Delta B_{i}, \Delta B_{d i}, \Delta C_{i}, \Delta G_{i}, \Delta G_{d i}, \Delta H_{i}, \Delta H_{d i}, \Delta D_{i}, \Delta \bar{A}_{i}, \Delta \bar{A}_{d i}, \Delta \bar{B}_{i}, \Delta \bar{B}_{d i}, \Delta \bar{C}_{i}, \Delta \bar{G}_{i}, \Delta \bar{G}_{d i}, \Delta \bar{H}_{i}$, $\Delta \bar{H}_{d i}, \Delta \bar{D}_{i}, \Delta L_{i}, \Delta J_{i}, \Delta B_{z i}, \Delta H_{z i}, \Delta K_{i}$ are unknown matrices representing time-varying parameter uncertainties, and the admissible uncertainties are assumed to be modeled in the form

$$
\left[\begin{array}{ccccc}
\Delta A_{i} & \Delta A_{d i} & \Delta B_{i} & \Delta B_{d i} & \Delta C_{i} \\
\Delta G_{i} & \Delta G_{d i} & \Delta H_{i} & \Delta H_{d i} & \Delta D_{i} \\
\Delta \bar{A}_{i} & \Delta \bar{A}_{d i} & \Delta \bar{B}_{i} & \Delta \bar{B}_{d i} & \Delta \bar{C}_{i} \\
\Delta \bar{G}_{i} & \Delta \bar{G}_{d i} & \Delta \bar{H}_{i} & \Delta \bar{H}_{d i} & \Delta \bar{D}_{i} \\
\Delta L_{i} & \Delta J_{i} & \Delta B_{z i} & \Delta H_{z i} & \Delta K_{i}
\end{array}\right]=\left[\begin{array}{c}
M_{i 1} \\
M_{i 2} \\
M_{i 3} \\
M_{i 4} \\
M_{i 5}
\end{array}\right] \Delta_{1}\left[\begin{array}{lllll}
N_{i 1} & N_{i 2} & N_{i 3} & N_{i 4} & N_{i 5}
\end{array}\right],
$$

where $M_{i 1}, M_{i 2}, M_{i 3}, M_{i 4}, M_{i 5}, N_{i 1}, N_{i 2}, N_{i 3}, N_{i 4}, N_{i 5}$ are known real constant matrices and $\Delta_{1}$ is the uncertain time-varying matrix satisfying $\Delta_{1}^{T} \Delta_{1} \leq I$.

The vector-valued nonlinear functions $f, f_{d}$, are assumed to satisfy the following sectorbounded conditions:

$$
\begin{aligned}
& {\left[f(x(t))-\hat{R}_{1} x(t)\right]^{T}\left[f(x(t))-\hat{R}_{2} x(t)\right] \leq 0, \quad \forall x(t) \in R^{n},} \\
& {\left[f_{d}(x(t-d(t)))-\hat{S}_{1} x(t-d(t))\right]^{T}\left[f_{d}(x(t-d(t)))-\hat{S}_{2} x(t-d(t))\right]} \\
& \quad \leq 0, \quad \forall x(t-d(t)) \in R^{n},
\end{aligned}
$$

where $\hat{R}_{1}, \hat{R}_{2}, \hat{S}_{1}, \hat{S}_{2} \in R^{n \times n}$ are known real constant matrices, and $\hat{R}=\hat{R}_{1}-\hat{R}_{2}, \hat{S}=\hat{S}_{1}-\hat{S}_{2}$ are symmetric positive definite matrices.

Remark 1 When $E$ is a unit matrix, $B_{i}=0, B_{d i}=0, H_{i}=0, H_{d i}=0, D_{i}=0, \bar{B}_{i}=0, \bar{B}_{d i}=0$, $\bar{H}_{i}=0, \bar{H}_{d i}=0, \bar{D}_{i}=0, J_{i}=0, B_{z i}=0, H_{z i}=0, K_{i}=0, \Delta B_{i}=0, \Delta B_{d i}=0, \Delta G_{i}=0, \Delta G_{d i}=0$, $\Delta H_{i}=0, \Delta H_{d i}=0, \Delta D_{i}=0, \Delta \bar{B}_{i}=0, \Delta \bar{B}_{d i}=0, \Delta \bar{G}_{i}=0, \Delta \bar{G}_{d i}=0, \Delta \bar{H}_{i}=0, \Delta \bar{H}_{d i}=0$, $\Delta \bar{D}_{i}=0, \Delta L_{i}=0, \Delta J_{i}=0, \Delta B_{z i}=0, \Delta H_{z i}=0, \Delta K_{i}=0$, system (1) was studied in [10]. The system in this paper is a class of stochastic time-delay systems broader than others. 
In this paper, we consider a full-order filter with the following form:

$$
\begin{aligned}
& \left\{\begin{array}{l}
E_{f i}(t) d \hat{x}(t)=A_{f i}(t) \hat{x}(t) d t+B_{f i}(t) d y(t)+C_{f i}(t) \hat{x}(t-d(t)) d t, \\
\hat{z}(t)=L_{f i}(t) \hat{x}(t)+J_{f i}(t) \hat{x}(t-d(t)),
\end{array}\right. \\
& E_{f i}(t)=E_{f i}+\Delta E_{f i}, \quad A_{f i}(t)=A_{f i}+\Delta A_{f i}, \quad B_{f i}(t)=B_{f i}+\Delta B_{f i}, \\
& C_{f i}(t)=C_{f i}+\Delta C_{f i}, \quad L_{f i}(t)=L_{f i}+\Delta L_{f i}, \quad J_{f i}(t)=J_{f i}+\Delta J_{f i},
\end{aligned}
$$

where $\hat{x}(t) \in R^{n}$ is the filter state and $\hat{z}(t) \in R^{q}$ is the estimated vector, $E_{f i}, A_{f i}, B_{f}, C_{f i}$, $L_{f i}, J_{f i}$ are the desired filter matrices to be designed, $E_{f i}$ may be a singular square matrix, $\Delta E_{f i}, \Delta A_{f i}, \Delta B_{f i}, \Delta C_{f i}, \Delta L_{f i}, \Delta J_{f i}$ are the filter gain variations with the following form:

$$
\left[\begin{array}{ccc}
\Delta E_{f i} & \Delta A_{f i} & \Delta B_{f i} \\
\Delta C_{f i} & \Delta L_{f i} & \Delta J_{f i}
\end{array}\right]=\left[\begin{array}{c}
M_{i 6} \\
M_{i 7}
\end{array}\right] \Delta_{2}\left[\begin{array}{lll}
N_{i 6} & N_{i 7} & N_{i 8}
\end{array}\right],
$$

where $M_{i 6}, M_{i 7}, N_{i 6}, N_{i 7}, N_{i 8}$ are known real constant matrices and $\Delta_{2}$ is unknown timevarying matrix function satisfying $\Delta_{2}^{T} \Delta_{2} \leq I$.

Remark 2 When $E_{f i}$ is a unit matrix, and $C_{f i}=0, J_{f i}=0, \Delta E_{f i}=0, \Delta C_{f i}=0, \Delta J_{f i}=0$, the filter (6) was studied in [9]. The objective in this paper improves the function of the filter.

Applying this filter to system (1), we obtain the following filtering error system:

$$
\left\{\begin{aligned}
E d x(t)= & {\left[A x(t)+A_{d} x(t-d(t))+B f(x(t))+B_{d} f_{d}(x(t-d(t)))+C v(t)\right] d t } \\
& +\left[G x(t)+G_{d} x(t-d(t))+H f(x(t))\right. \\
& \left.+H_{d} f_{d}(x(t-d(t)))+D v(t)\right] d \omega(t), \\
\tilde{E} d \tilde{x}(t)= & {\left[\tilde{A} \tilde{x}(t)+\tilde{A}_{d} \tilde{x}(t-d(t))+\tilde{B} f(x(t))+\tilde{B}_{d} f_{d}(x(t-d(t)))+\tilde{C} v(t)\right] d t } \\
& +\left[\tilde{G} W \tilde{x}(t)+\tilde{G}_{d} W \tilde{x}(t-d(t))+\tilde{H} f(x(t))\right. \\
& \left.+\tilde{H}_{d} f_{d}(x(t-d(t)))+\tilde{D} v(t)\right] d \omega(t), \\
e(t)=\tilde{L} \tilde{x}(t) & +\tilde{J} \tilde{x}(t-d(t))+\tilde{B}_{z i} f(x(t))+\tilde{H}_{z i} f(x(t-d(t)))+\tilde{K} v(t),
\end{aligned}\right.
$$

where

$$
\begin{aligned}
& \tilde{x}(t)=\left[x^{T}(t),(x(t)-\hat{x}(t))^{T}\right]^{T}, \quad e(t)=z(t)-\hat{z}(t), \\
& \tilde{E}=\tilde{E}_{i}+\Delta \tilde{E}_{i}, \quad A=A_{i}+\Delta A_{i}, \quad A_{d}=A_{d i}+\Delta A_{d i}, \\
& B=B_{i}+\Delta B_{i}, \quad B_{d}=B_{d i}+\Delta B_{d i}, \quad C=C_{i}+\Delta C_{i}, \\
& G=G_{i}+\Delta G_{i}, \quad G_{d}=G_{d i}+\Delta G_{d i}, \quad H=H_{i}+\Delta H_{i}, \\
& H_{d}=H_{d i}+\Delta H_{d i}, \quad D=D_{i}+\Delta D_{i}, \quad \tilde{A}=\tilde{A}_{i}+\Delta \tilde{A}_{i}, \\
& \tilde{A}_{d}=\tilde{A}_{d i}+\Delta \tilde{A}_{d i}, \quad \tilde{B}=\tilde{B}_{i}+\Delta \tilde{B}_{i}, \quad \tilde{B}_{d}=\tilde{B}_{d i}+\Delta \tilde{B}_{d i}, \\
& \tilde{C}=\tilde{C}_{i}+\Delta \tilde{C}_{i}, \quad \tilde{G}=\tilde{G}_{i}+\Delta \tilde{G}_{i}, \quad \tilde{G}_{d}=\tilde{G}_{d i}+\Delta \tilde{G}_{d i},
\end{aligned}
$$




$$
\begin{aligned}
& \tilde{H}=\tilde{H}_{i}+\Delta \tilde{H}_{i}, \quad \tilde{H}_{d}=\tilde{H}_{d i}+\Delta \tilde{H}_{d i}, \quad \tilde{D}=\tilde{D}_{i}+\Delta \tilde{D}_{i}, \\
& \tilde{L}=\tilde{L}_{i}+\Delta \tilde{L}_{i}, \quad \tilde{J}=\tilde{J}_{i}+\Delta \tilde{J}_{i}, \quad \tilde{B}_{z i}=B_{z i}+\Delta B_{z i}, \\
& \tilde{H}_{z i}=H_{z i}+\Delta H_{z i}, \quad \tilde{K}=K_{i}+\Delta K_{i}, \\
& \tilde{E}_{i}=\left[\begin{array}{cc}
E & 0 \\
0 & E_{f i}
\end{array}\right], \quad \tilde{A}_{i}=\left[\begin{array}{cc}
A_{i} & 0 \\
A_{i}-A_{f i}-B_{f i} \bar{A}_{i} & A_{f i}
\end{array}\right], \\
& \tilde{A}_{d i}=\left[\begin{array}{cc}
A_{d i} & 0 \\
A_{d i}-C_{f i}-B_{f i} \bar{A}_{d i} & C_{f i}
\end{array}\right] \text {, } \\
& \tilde{B}_{i}=\left[\begin{array}{c}
B_{i} \\
B_{i}-B_{f i} \bar{B}_{i}
\end{array}\right], \quad \tilde{B}_{d i}=\left[\begin{array}{c}
B_{d i} \\
B_{d i}-B_{f i} \bar{B}_{d i}
\end{array}\right] \text {, } \\
& \tilde{C}_{i}=\left[\begin{array}{c}
C_{i} \\
C_{i}-B_{f_{i}} \bar{C}_{i}
\end{array}\right], \quad \tilde{G}_{i}=\left[\begin{array}{c}
G_{i} \\
G_{i}-B_{f i} \bar{G}_{i}
\end{array}\right] \text {, } \\
& \tilde{G}_{d i}=\left[\begin{array}{c}
G_{d i} \\
G_{d i}-B_{f i} \bar{G}_{d i}
\end{array}\right], \quad \tilde{H}_{i}=\left[\begin{array}{c}
H_{i} \\
H_{i}-B_{f i} \bar{H}_{i}
\end{array}\right] \text {, } \\
& \tilde{H}_{d i}=\left[\begin{array}{c}
H_{d i} \\
H_{d i}-B_{f i} \bar{H}_{d i}
\end{array}\right], \quad \tilde{D}_{i}=\left[\begin{array}{c}
D_{i} \\
D_{i}-B_{f i} \bar{D}_{i}
\end{array}\right] \text {, } \\
& \tilde{L}_{i}=\left[\begin{array}{ll}
L_{i}-L_{f i} & L_{f i}
\end{array}\right], \quad \tilde{J}_{i}=\left[\begin{array}{ll}
J_{i}-J_{f i} & J_{f i}
\end{array}\right], \quad W=\left[\begin{array}{ll}
I & 0
\end{array}\right], \\
& \Delta \tilde{E}_{i}=\left[\begin{array}{cc}
0 & 0 \\
0 & \Delta E_{f i}
\end{array}\right], \quad \Delta \tilde{A}_{i}=\left[\begin{array}{cc}
\Delta A_{i} & 0 \\
\Delta A_{i}-\Delta A_{f i}-\Delta B_{f i} \bar{A}_{i}-B_{f i} \Delta \bar{A}_{i} & \Delta A_{f i}
\end{array}\right], \\
& \Delta \tilde{A}_{d i}=\left[\begin{array}{cc}
\Delta A_{d i} & 0 \\
\Delta A_{d i}-\Delta C_{f i}-\Delta B_{f i} \bar{A}_{d i}-B_{f i} \Delta \bar{A}_{d i} & \Delta C_{f i}
\end{array}\right] \text {, } \\
& \Delta \tilde{B}_{i}=\left[\begin{array}{c}
\Delta B_{i} \\
\Delta B_{i}-\Delta B_{f i} \bar{B}_{i}-B_{f i} \Delta \bar{B}_{i}
\end{array}\right], \quad \Delta \tilde{B}_{d i}=\left[\begin{array}{c}
\Delta B_{d i} \\
\Delta B_{d i}-\Delta B_{f i} \bar{B}_{d i}-B_{f i} \Delta \bar{B}_{d i}
\end{array}\right] \text {, } \\
& \Delta \tilde{C}_{i}=\left[\begin{array}{c}
\Delta C_{i} \\
\Delta C_{i}-\Delta B_{f i} \bar{C}_{i}-B_{f i} \Delta \bar{C}_{i}
\end{array}\right], \quad \Delta \tilde{G}_{i}=\left[\begin{array}{c}
\Delta G_{i} \\
\Delta G_{i}-\Delta B_{f i} \bar{G}-B_{f i} \Delta \bar{G}_{i}
\end{array}\right], \\
& \Delta \tilde{G}_{d i}=\left[\begin{array}{c}
\Delta G_{d i} \\
\Delta G_{d i}-\Delta B_{f i} \bar{G}_{d i}-B_{f i} \Delta \bar{G}_{d i}
\end{array}\right], \quad \Delta \tilde{H}_{i}=\left[\begin{array}{c}
\Delta H_{i} \\
\Delta H_{i}-\Delta B_{f i} \bar{H}_{i}-B_{f i} \Delta \bar{H}_{i}
\end{array}\right] \text {, } \\
& \Delta \tilde{H}_{d i}=\left[\begin{array}{c}
\Delta H_{d i} \\
\Delta H_{d i}-\Delta B_{f i} \bar{H}_{d i}-B_{f i} \Delta \bar{H}_{d i}
\end{array}\right], \quad \Delta \tilde{D}_{i}=\left[\begin{array}{c}
\Delta D_{i} \\
\Delta D_{i}-\Delta B_{f i} \bar{D}_{i}-B_{f i} \Delta \bar{D}_{i}
\end{array}\right] \text {, } \\
& \Delta \tilde{L}_{i}=\left[\begin{array}{ll}
\Delta L_{i}-\Delta L_{f i} & \Delta L_{f i}
\end{array}\right], \quad \Delta \tilde{J}_{i}=\left[\begin{array}{ll}
\Delta J_{i}-\Delta J_{f i} & \Delta J_{f i}
\end{array}\right] .
\end{aligned}
$$

Denote $\langle x, H y\rangle_{T}=\int_{0}^{T} x^{T}(t) H y(t) d t$, in this article, we focus our attention on the quadratic supply rate

$$
E(v, e, T)=\langle e, Q e\rangle_{T}+2\langle e, S v\rangle_{T}+\langle v, R v\rangle_{T},
$$

where $Q, S, R$ are appropriately dimensioned, and $Q, R$ are symmetric matrices. 
Definition 1 [18] If filtering error system (9) is asymptotically stable and for all $T>0$ and $0 \neq v(t) \in L_{2}[0, \infty)$, there exists $\alpha>0$ such that the following inequality is well defined, then

$$
E(v, e, T)>\alpha\langle v, v\rangle_{T}
$$

Definition 2 Given a set of suited dimension real matrices $Q, S, R$, where $Q, R$ are symmetric matrices, system (6) is called a robust dissipative filter of uncertain system (1) if there exist $A_{f i}, B_{f i}, C_{f i}, L_{f i}, J_{f i}, E_{f i}$ such that

(a) the augmented system (9) with $v(t)=0$ is robust asymptotically stable for all uncertainties;

(b) the filtering error system is strict robust $(Q, S, R)$ dissipative.

Our aim is to determine parameters $A_{f i}, B_{f i}, C_{f i}, L_{f i}, J_{f i}, E_{f i}$ such that system (6) is a robust dissipative filter for uncertain system (1).

Lemma 1 [8] Given a set of suited dimension real matrices $Q, H, E, Q$ is a symmetric matrix, such that

$$
Q+H F E+E^{T} F^{T} H^{T}<0
$$

for all $F$ satisfies $F^{T} F \leq I$ if and only if there exists $\varepsilon>0$ such that

$$
Q+\varepsilon H H^{T}+\varepsilon^{-1} E^{T} E<0 \text {. }
$$

Lemma 2 (Schur complement) Given a symmetric matrix $S=\left[\begin{array}{ll}S_{11} & S_{12} \\ S_{12}^{T} & S_{22}\end{array}\right]$, where $S_{11} \in R^{r \times r}$, the following three conditions are equivalent:

(i) $S<0$;

(ii) $S_{11}<0, S_{22}-S_{12}^{T} S_{11}^{-1} S_{12}<0$;

(iii) $S_{22}<0, S_{11}-S_{12} S_{22}^{-1} S_{12}^{T}<0$.

\section{Main results}

Theorem 1 Given matrices $Q, S, R$, where $Q, R$ are symmetric and $Q$ is negative definite and with all uncertainties, the robust dissipative filtering problem is solved for the uncertain Markovian jump system (9) if there exist symmetric matrices $P_{l}>0, P_{i}>0, U>0, U_{i}>$ 0 , and $\tilde{U}>0$, positive scalars $\lambda_{1}, \lambda_{2}$, and any appropriately dimensioned matrices $N_{i}=$ $\left[\bar{N}_{1 i}^{T} \bar{N}_{2 i}^{T}\right]^{T}, M_{i}=\left[\bar{M}_{1 i}^{T} \bar{M}_{2 i}^{T}\right]^{T}, X=X^{T}=\left[\begin{array}{cc}X_{11} & X_{12} \\ * & X_{22}\end{array}\right] \geq 0$, and under zero conditions for nonzero $v(t) \in L_{2}(0, \infty)$, such that the following LMI holds:

$$
\begin{aligned}
& E^{T} P_{l}=P_{l} E \geq 0, \\
& \tilde{E}^{T} P_{i}=P_{i} \tilde{E} \geq 0,
\end{aligned}
$$




$$
\Omega_{i}=\left[\begin{array}{ccccccccccccc}
\Omega_{11 i} & P_{l} A_{d} & 0 & 0 & 0 & \Omega_{16 i} & P_{l} B_{d} & P_{l} C & 0 & 0 & 0 & G^{T} P_{l} & 0 \\
* & -\lambda_{2} \breve{S}_{1} & 0 & 0 & 0 & 0 & -\lambda_{2} \breve{S}_{2} & 0 & 0 & 0 & 0 & G_{d}^{T} P_{l} & 0 \\
* & * & \Omega_{33 i} & \Omega_{34 i} & -M_{1 i} & P_{i} \tilde{B} & P_{i} \tilde{B}_{d} & \Omega_{38 i} & 0 & 0 & \tilde{L}^{T} Q & 0 & \Omega_{313 i} \\
* & * & * & \Omega_{44 i} & -M_{2 i} & 0 & 0 & -\tilde{J}^{T} S & 0 & 0 & \tilde{J}^{T} Q & 0 & \Omega_{413 i} \\
* & * & * & * & -U_{i} & 0 & 0 & 0 & 0 & 0 & 0 & 0 & 0 \\
* & * & * & * & * & -\lambda_{1} I & 0 & -\tilde{B}_{z i}^{T} S & 0 & 0 & \tilde{B}_{z i}^{T} Q & H^{T} P_{l} & \tilde{H}^{T} P_{i} \\
* & * & * & * & * & * & -\lambda_{2} I & -\tilde{H}_{z i}^{T} S & 0 & 0 & \tilde{H}_{z i}^{T} Q & H_{d}^{T} P_{l} & \tilde{H}_{d}^{T} P_{i} \\
* & * & * & * & * & * & * & \Omega_{88 i} & 0 & 0 & \tilde{K}^{T} Q & D^{T} P_{l} & \tilde{D}^{T} P_{i} \\
* & * & * & * & * & * & * & * & -\psi_{1} & 0 & 0 & 0 & 0 \\
* & * & * & * & * & * & * & * & * & -\psi_{2} & 0 & 0 & 0 \\
* & * & * & * & * & * & * & * & * & * & Q & 0 & 0 \\
* & * & * & * & * & * & * & * & * & * & * & -P_{l} & 0 \\
* & * & * & * & * & * & * & * & * & * & * & * & -P_{i}
\end{array}\right]
$$

where

$$
\begin{aligned}
& \Omega_{11 i}=\sum_{l=1}^{N} \pi_{i l} E^{T} P_{l}+P_{l} A+A^{T} P_{l}-\lambda_{1} \breve{R}_{1}, \quad \Omega_{16 i}=-\lambda_{1} \breve{R}_{2}+P_{l} B \\
& \Omega_{33 i}=\sum_{j=1}^{N} \pi_{i j} \tilde{E}^{T} P_{j}+W^{T} U W+U_{i}+d_{1} W^{T} \tilde{U} W+P_{i} \tilde{A}+\tilde{A}^{T} P_{i}+d_{1} X_{11}+\bar{N}_{1 i}+\bar{N}_{1 i}^{T}, \\
& \Omega_{34 i}=P_{i} \tilde{A}_{d}+d_{1} X_{12}-\bar{N}_{1 i}+\bar{N}_{2 i}^{T}+\bar{M}_{1 i}, \quad \Omega_{38 i}=P_{i} \tilde{C}-\tilde{L}^{T} S, \quad \Omega_{313 i}=W^{T} \tilde{G}^{T} P_{i}, \\
& \Omega_{44 i}=-\left(1-h_{i}\right) W^{T} U W+d_{1} X_{22}-\bar{N}_{2 i}-\bar{N}_{2 i}^{T}+\bar{M}_{2 i}^{T}+\bar{M}_{2 i}, \quad \Omega_{413 i}=W^{T} \tilde{G}_{d}^{T} P_{i}, \\
& \Omega_{88 i}=-S^{T} \tilde{K}-\tilde{K}^{T} S-R, \\
& \psi_{1}=\left[\begin{array}{cccc}
X_{11} & X_{12} & 0 & \bar{N}_{1 i} \\
* & X_{22} & 0 & \bar{N}_{2 i} \\
* & * & W^{T} \tilde{U} W & 0 \\
* & * & * & 0
\end{array}\right], \quad \psi_{2}=\left[\begin{array}{cccc}
X_{11} & X_{12} & 0 & \bar{M}_{1 i} \\
* & X_{22} & 0 & \bar{M}_{2 i} \\
* & * & W^{T} \tilde{U} W & 0 \\
* & * & * & 0
\end{array}\right] .
\end{aligned}
$$

Proof For each $r(t)=i \in S$, choose a Lyapunov-Krasovskii functional for system (9) as

$$
V(x, \tilde{x}, t, i)=V_{1}(x, \tilde{x}, t, i)+V_{2}(x, \tilde{x}, t, i)+V_{3}(x, \tilde{x}, t, i)+V_{4}(x, \tilde{x}, t, i)+V_{5}(x, \tilde{x}, t, i)
$$

where

$$
\begin{aligned}
& V_{1}(x, \tilde{x}, t, i)=x^{T}(t) E^{T} P_{l} x(t), \\
& V_{2}(x, \tilde{x}, t, i)=\tilde{x}^{T}(t) \tilde{E}^{T} P_{i} \tilde{x}(t), \\
& V_{3}(x, \tilde{x}, t, i)=\int_{t-d(t)}^{t} \tilde{x}^{T}(s) W^{T} U W \tilde{x}(s) d s, \\
& V_{4}(x, \tilde{x}, t, i)=\int_{t-d_{1}}^{t} \tilde{x}^{T}(s) U_{i} \tilde{x}(s) d s, \\
& V_{5}(x, \tilde{x}, t, i)=\int_{-d_{1}}^{0} \int_{t+\theta}^{t} \tilde{x}^{T}(s) W^{T} \tilde{U} W \tilde{x}(s) d s d \theta,
\end{aligned}
$$

and $P_{l}>0, P_{i}>0, U>0, U_{i}>0, \tilde{U}>0 . V_{3}(x, \tilde{x}, t, i), V_{4}(x, \tilde{x}, t, i), V_{5}(x, \tilde{x}, t, i)$ are governed by $t$, so we have $V_{\tilde{x} \tilde{x}}(\tilde{x}, t, i)=P_{l}+P_{i}$. 
When $v(t) \equiv 0$,

$$
\begin{aligned}
& L V_{1}(x, \tilde{x}, t, i)= x^{T}(t) \sum_{l=1}^{N} \pi_{i l} E^{T} P_{l} x(t) \\
&+2 x^{T}(t) P_{l}\left[A x(t)+A_{d} x(t-d(t))+B f(x(t))+B_{d} f_{d}(x(t-d(t)))\right] \\
&+\left\{\left[G x(t)+G_{d} x(t-d(t))+H f(x(t))+H_{d} f_{d}(x(t-d(t)))\right]^{T}\right. \\
&\left.\times P_{l}\left[G x(t)+G_{d} x(t-d(t))+H f(x(t))+H_{d} f_{d}(x(t-d(t)))\right]\right\}, \\
& L V_{2}(x, \tilde{x}, t, i)=\tilde{x}^{T}(t) \sum_{j=1}^{N} \pi_{i j} \tilde{E}^{T} P_{j} \tilde{x}(t) \\
&+2 \tilde{x}^{T}(t) P_{i}\left[\tilde{x} \tilde{x}(t)+\tilde{A} d \tilde{x}(t-d(t))+\tilde{B} f(x(t))+\tilde{B}_{d} f_{d}(x(t-d(t)))\right] \\
&+\left\{\left[\tilde{G} W \tilde{x}(t)+\tilde{G}_{d} W \tilde{x}(t-d(t))+\tilde{H} f(x(t))+\tilde{H}_{d} f_{d}(x(t-d(t)))\right]^{T}\right. \\
&\left.\times P_{i}\left[\tilde{G} W \tilde{x}(t)+\tilde{G}_{d} W \tilde{x}(t-d(t))+\tilde{H} f(x(t))+\tilde{H}_{d} f_{d}(x(t-d(t)))\right]\right\}, \\
& L V_{3}(x, \tilde{x}, t, i)= \tilde{x}^{T}(t) W^{T} U W \tilde{x}(t)-(1-\dot{d}(t)) \tilde{x}^{T}(t-d(t)) W^{T} U W \tilde{x}(t-d(t)), \\
& L V_{4}(x, \tilde{x}, t, i)= \tilde{x}^{T}(t) U_{i} \tilde{x}(t)-\tilde{x}^{T}\left(t-d_{1}\right) U_{i} \tilde{x}\left(t-d_{1}\right), \\
& L V_{5}(x, \tilde{x}, t, i)= d_{1} \tilde{x}^{T}(t) W^{T} \tilde{U} W \tilde{x}(t)-\int_{t-d(t)}^{t} \tilde{x}^{T}(s) W W^{T} \tilde{U} W \tilde{x}(s) d s \\
&-\int_{t-d_{1}}^{t-d(t)} \tilde{x} \tilde{x}^{T}(s) W^{T} \tilde{U} W \tilde{x}(s) d s .
\end{aligned}
$$

Using the Newton-Leibniz formula, for any appropriately dimensioned matrices $N_{i}, M_{i}$, the following equations are true:

$$
\begin{aligned}
& 0=2 \xi_{1}^{T}(t) N_{i}\left[\tilde{x}(t)-\tilde{x}(t-d(t))-\int_{t-d(t)}^{t} \dot{\tilde{x}}(s) d s\right] \\
& 0=2 \xi_{1}^{T}(t) M_{i}\left[\tilde{x}(t-d(t))-\tilde{x}\left(t-d_{1}\right)-\int_{t-d_{1}}^{t-d(t)} \dot{\tilde{x}}(s) d s\right]
\end{aligned}
$$

where $\xi_{1}(t)=\left[\tilde{x}^{T}(t) \tilde{x}^{T}(t-d(t))\right]^{T}$.

On the other hand, for any semi-positive definite matrix $X=X^{T}=\left[\begin{array}{cc}X_{11} & X_{12} \\ * & X_{22}\end{array}\right] \geq 0$, the following equation holds:

$$
\begin{aligned}
0 & =\int_{t-d_{1}}^{t} \xi_{1}^{T}(t) X \xi_{1}(t) d s-\int_{t-d_{1}}^{t} \xi_{1}^{T}(t) X \xi_{1}(t) d s \\
& =d_{1} \xi_{1}^{T}(t) X \xi_{1}(t)-\int_{t-d(t)}^{t} \xi_{1}^{T}(t) X \xi_{1}(t) d s-\int_{t-d_{1}}^{t-d(t)} \xi_{1}^{T}(t) X \xi_{1}(t) d s
\end{aligned}
$$

In addition, it is clear that the following equation is also true:

$$
\begin{aligned}
\int_{t-d_{1}}^{t} \xi_{1}^{T}(t) W^{T} \tilde{U} W \xi_{1}(t) d s= & \int_{t-d(t)}^{t} \xi_{1}^{T}(t) W^{T} \tilde{U} W \xi_{1}(t) d s \\
& +\int_{t-d_{1}}^{t-d(t)} \xi_{1}^{T}(t) W^{T} \tilde{U} W \xi_{1}(t) d s
\end{aligned}
$$


Notice that (5) implies

$$
\begin{aligned}
& {\left[\begin{array}{c}
x(t) \\
f(x(t))
\end{array}\right]^{T}\left[\begin{array}{cc}
\breve{R}_{1} & \breve{R}_{2} \\
\breve{R}_{2}^{T} & I
\end{array}\right]\left[\begin{array}{c}
x(t) \\
f(x(t))
\end{array}\right] \leq 0,} \\
& {\left[\begin{array}{c}
x(t-d(t)) \\
f_{d}(x(t-d(t)))
\end{array}\right]^{T}\left[\begin{array}{cc}
\breve{S}_{1} & \breve{S}_{2} \\
\breve{S}_{2}^{T} & I
\end{array}\right]\left[\begin{array}{c}
x(t-d(t)) \\
f_{d}(x(t-d(t)))
\end{array}\right] \leq 0,}
\end{aligned}
$$

where $\breve{R}_{1}=\left(\hat{R}_{1}^{T} \hat{R}_{2}+\hat{R}_{2}^{T} \hat{R}_{1}\right) / 2, \breve{R}_{2}=-\left(\hat{R}_{1}^{T}+\hat{R}_{2}^{T}\right) / 2, \breve{S}_{1}=\left(\hat{S}_{1}^{T} \hat{S}_{2}+\hat{S}_{2}^{T} \hat{S}_{1}\right) / 2, \breve{S}_{2}=-\left(\hat{S}_{1}^{T}+\hat{S}_{2}^{T}\right) / 2$.

Then, adding the terms on the right of Eqs. (14)-(16) and (18) to $V(x, \tilde{x}, t, i)$ yields

$$
\begin{aligned}
& L V(x, \tilde{x}, t, i) \\
& \leq L V(x, \tilde{x}, t, i)-\lambda_{1}\left[\begin{array}{c}
x(t) \\
f(x(t))
\end{array}\right]^{T}\left[\begin{array}{cc}
\breve{R}_{1} & \breve{R}_{2} \\
\breve{R}_{2}^{T} & I
\end{array}\right]\left[\begin{array}{c}
x(t) \\
f(x(t))
\end{array}\right] \\
& \quad-\lambda_{2}\left[\begin{array}{c}
x(t-d(t)) \\
f_{d}(x(t-d(t)))
\end{array}\right]^{T}\left[\begin{array}{cc}
\breve{S}_{1} & \breve{S}_{2} \\
\breve{S}_{2}^{T} & I
\end{array}\right]\left[\begin{array}{c}
x(t-d(t)) \\
f_{d}(x(t-d(t)))
\end{array}\right] \\
& \quad+2 \xi_{1}^{T}(t) N_{i}\left[\tilde{x}(t)-\tilde{x}(t-d(t))-\int_{t-d(t)}^{t} \dot{\tilde{x}}(s) d s\right] \\
& \quad+2 \xi_{1}^{T}(t) M_{i}\left[\tilde{x}(t-d(t))-\tilde{x}\left(t-d_{1}\right)-\int_{t-d_{1}}^{t-d(t)} \dot{\tilde{x}}(s) d s\right] \\
& \quad+d_{1} \xi_{1}^{T}(t) X \xi_{1}(t)-\int_{t-d(t)}^{t} \xi_{1}^{T}(t) X \xi_{1}(t) d s-\int_{t-d_{1}}^{t-d(t)} \xi_{1}^{T}(t) X \xi_{1}(t) d s \\
& \leq \xi^{T}(t) H_{i} \xi(t),
\end{aligned}
$$

where

$$
\begin{aligned}
& \xi(t)=\left[\begin{array}{lllllll}
x^{T}(t) & x^{T}(t-d(t)) & \tilde{x}^{T}(t) & \tilde{x}^{T}(t-d(t)) & \tilde{x}^{T}\left(t-d_{1}\right) & f(x(t)) & f_{d}(x(t-d(t)))
\end{array}\right. \\
& \left.\int_{t-d(t)}^{t} \eta^{T}(t, s) d s \quad \int_{t-d_{1}}^{t-d(t)} \eta^{T}(t, s) d s\right]^{T} \\
& \eta(t, s)=\left[\begin{array}{lll}
\xi_{1}^{T}(t) & \tilde{x}^{T}(s) & \dot{\tilde{x}}^{T}(s)
\end{array}\right]^{T}, \\
& H_{i}=\left[\begin{array}{ccccccccc}
H_{11 i} & H_{12 i} & 0 & 0 & 0 & H_{16 i} & P_{l} B_{d}+G^{T} P_{l} H_{d} & 0 & 0 \\
* & H_{22 i} & 0 & 0 & 0 & G_{d}^{T} P_{l} H & -\lambda_{2} \breve{S}_{2}+G_{d}^{T} P_{l} H_{d} & 0 & 0 \\
* & * & H_{33 i} & H_{34 i} & -\bar{M}_{1 i} & H_{36 i} & P_{i} \tilde{B}_{d}+W^{T} \tilde{G}^{T} P_{i} \tilde{H}_{d} & 0 & 0 \\
* & * & * & H_{44 i} & -\bar{M}_{2 i} & W^{T} \tilde{G}_{d}^{T} P_{i} \tilde{H} & W^{T} \tilde{G}_{d}^{T} P_{i} \tilde{H}_{d} & 0 & 0 \\
* & * & * & * & -U_{i} & 0 & 0 & 0 & 0 \\
* & * & * & * & * & H_{66 i} & H_{67 i} & 0 & 0 \\
* & * & * & * & * & * & H_{77 i} & 0 & 0 \\
* & * & * & * & * & * & * & -\psi_{1} & 0 \\
* & * & * & * & * & * & * & * & -\psi_{2}
\end{array}\right], \\
& H_{11 i}=\sum_{l=1}^{N} \pi_{i l} E^{T} P_{l}+P_{l} A+A^{T} P_{l}+G^{T} P_{l} G-\lambda_{1} \breve{R}_{1}, \quad H_{12 i}=P_{l} A_{d}+G^{T} P_{l} G_{d}
\end{aligned}
$$




$$
\begin{aligned}
& H_{16 i}=-\lambda_{1} \breve{R}_{2}+P_{l} B+G^{T} P_{l} H, \quad H_{22 i}=-\lambda_{2} \breve{S}_{1}+G_{d}^{T} P_{l} G_{d}, \\
& H_{33 i}=\sum_{j=1}^{N} \pi_{i j} \tilde{E}^{T} P_{j}+W^{T} U W+U_{i}+d_{1} W^{T} \tilde{U} W+P_{i} \tilde{A} \\
& +\tilde{A}^{T} P_{i}+W^{T} \tilde{G}^{T} P_{i} \tilde{G} W+d_{1} X_{11}+\bar{N}_{1 i}+\bar{N}_{1 i}^{T}, \\
& H_{34 i}=P_{i} \tilde{A}_{d}+W^{T} \tilde{G}^{T} P_{i} \tilde{G}_{d} W+d_{1} X_{12}-\bar{N}_{1 i}+\bar{N}_{2 i}^{T}+\bar{M}_{1 i}, \\
& H_{36 i}=P_{i} \tilde{B}+W^{T} \tilde{G}^{T} P_{i} \tilde{H}, \\
& H_{44 i}=-\left(1-h_{i}\right) W^{T} U W+W^{T} \tilde{G}_{d}^{T} P_{i} \tilde{G}_{d} W+d_{1} X_{22}-\bar{N}_{2 i}-\bar{N}_{2 i}^{T}+\bar{M}_{2 i}^{T}+\bar{M}_{2 i} \text {, } \\
& H_{66 i}=\tilde{H}^{T} P_{i} \tilde{H}-\lambda_{1} I+H^{T} P_{l} H, \quad H_{67 i}=\tilde{H}^{T} P_{i} \tilde{H}_{d}+H^{T} P_{l} H_{d}, \\
& H_{77 i}=\tilde{H}_{d}^{T} P_{i} \tilde{H}_{d}-\lambda_{2} I+H_{d}^{T} P_{l} H_{d}, \\
& \psi_{1}=\left[\begin{array}{cccc}
X_{11} & X_{12} & 0 & \bar{N}_{1 i} \\
* & X_{22} & 0 & \bar{N}_{2 i} \\
* & * & W^{T} \tilde{U} W & 0 \\
* & * & * & 0
\end{array}\right], \quad \psi_{2}=\left[\begin{array}{cccc}
X_{11} & X_{12} & 0 & \bar{M}_{1 i} \\
* & X_{22} & 0 & \bar{M}_{2 i} \\
* & * & W^{T} \tilde{U} W & 0 \\
* & * & * & 0
\end{array}\right] .
\end{aligned}
$$

Via Lyapunov-Krasovskii, according to (13), if $H_{i}<0$ and $L V(x, \tilde{x}, t, i)<0$, system (9) is robust asymptotically stable.

When $v(t) \neq 0$,

$$
L V(x, \tilde{x}, v, t, i) \leq \varsigma(t)^{T}(t) H_{i}^{\prime} \varsigma(t)
$$

where

$$
\begin{aligned}
& \varsigma(t)=\left[\begin{array}{llllll}
x^{T}(t) & x^{T}(t-d(t)) & \tilde{x}^{T}(t) & \tilde{x}^{T}(t-d(t)) & \tilde{x}^{T}\left(t-d_{1}\right) & f(x(t))
\end{array}\right. \\
& \left.f_{d}(x(t-d(t))) \quad v^{T}(t) \quad \int_{t-d(t)}^{t} \eta^{T}(t, s) d s \quad \int_{t-d_{1}}^{t-d(t)} \eta^{T}(t, s) d s\right]^{T}, \\
& H_{i}^{\prime}=\left[\begin{array}{ccccccccc}
H_{11 i} & H_{12 i} & 0 & 0 & 0 & H_{16 i} & P_{l} B_{d}+G^{T} P_{l} H_{d} & H_{18 i}^{\prime} & 0 \\
* & H_{22 i} & 0 & 0 & 0 & G_{d}^{T} P_{l} H & -\lambda_{2} \breve{S}_{2}+G_{d}^{T} P_{l} H_{d} & G_{d}^{T} P_{l} D & 0 \\
* & * & H_{33 i} & H_{34 i} & -\bar{M}_{1 i} & H_{36 i} & P_{i} \tilde{B}_{d}+W^{T} \tilde{G}^{T} P_{i} \tilde{H}_{d} & H_{38 i}^{\prime} & 0 \\
* & * & * & H_{44 i} & -\bar{M}_{2 i} & W^{T} \tilde{G}_{d}^{T} P_{i} \tilde{H} & W^{T} \tilde{G}_{d}^{T} P_{i} \tilde{H}_{d} & H_{48 i}^{\prime} & 0 \\
* & * & * & * & -U_{i} & 0 & 0 & 0 & 0 \\
* & * & * & * & * & H_{66 i} & H_{67 i} & H_{68 i}^{\prime} & 0 \\
* & * & * & * & * & * & H_{77 i} & H_{78 i}^{\prime} & 0 \\
* & * & * & * & * & * & * & H_{88 i}^{\prime} & 0 \\
* & * & * & * & * & * & * & * & -\psi
\end{array}\right] \\
& H_{18 i}^{\prime}=P_{l} C+G^{T} P_{l} D, \quad H_{38 i}^{\prime}=P_{i} \tilde{C}+W^{T} \tilde{G}^{T} P_{i} \tilde{D}, \\
& H_{48 i}^{\prime}=W^{T} \tilde{G}_{d}^{T} P_{i} \tilde{D}, \quad H_{68 i}^{\prime}=\tilde{H}^{T} P_{i} \tilde{D}+H^{T} P_{l} D, \\
& H_{78 i}^{\prime}=\tilde{H}_{d}^{T} P_{i} \tilde{D}+H_{d}^{T} P_{l} D, \quad H_{88 i}^{\prime}=\tilde{D}^{T} P_{i} \tilde{D}+D^{T} P_{l} D, \\
& \psi=\left[\begin{array}{ll}
\psi_{1} & \\
& \psi_{2}
\end{array}\right] \text {. }
\end{aligned}
$$


Furthermore, we consider the dissipation performance. For the zero initial condition

$$
J(T, \alpha)=\int_{0}^{T}\left[e^{T}(t) Q e(t)+2 e^{T}(t) S v(t)+v^{T}(t)(R-\alpha I) v(t)\right] d t,
$$

it is easy to get $J(T, \alpha)=E(v, e, T)-\alpha\langle v, v\rangle_{T}$.

Consider the augmented system (9) is strict robust $(Q, S, R)$ dissipative if and only if there exists $\alpha>0$, with all $T>0$ and under zero conditions for nonzero $v(t) \in L_{2}[0, \infty)$, such that $J(T, \alpha) \geq 0$.

$$
\begin{aligned}
& \varsigma^{T}(t) H_{i}^{\prime} \varsigma(t)-J(T, \alpha) \\
& \quad=\int_{0}^{T}\left[\xi^{T}(t) H_{i} \xi(t)-e^{T}(t) Q e(t)-2 e^{T}(t) S v(t)-v^{T}(t)(R-\alpha I) v(t)\right] d t \\
& \quad=\int_{0}^{T}\left[\varsigma^{T}(t)\left(\Upsilon_{i}+\alpha \operatorname{diag}(0,0,0,0,0,0,0, I, 0,0)\right) \varsigma(t)\right] d t,
\end{aligned}
$$

where

$$
\begin{aligned}
& \Upsilon_{i}=\left[\begin{array}{ccccccccc}
\Upsilon_{11 i} & P_{l} A_{d}+G^{T} P_{l} G_{d} & 0 & 0 & 0 & \Upsilon_{16 i} & \Upsilon_{17 i} & \Upsilon_{18 i} & 0 \\
* & -\lambda_{2} \breve{S}_{1}+G_{d}^{T} P_{l} G_{d} & 0 & 0 & 0 & G_{d}^{T} P_{l} H & \Upsilon_{27 i} & G_{d}^{T} P_{l} D & 0 \\
* & * & \Upsilon_{33 i} & \Upsilon_{34 i} & -\bar{M}_{1 i} & \Upsilon_{36 i} & \Upsilon_{37 i} & \Upsilon_{38 i} & 0 \\
* & * & * & \Upsilon_{44 i} & -\bar{M}_{2 i} & \Upsilon_{46 i} & \Upsilon_{47 i} & \Upsilon_{48 i} & 0 \\
* & * & * & * & -U_{i} & 0 & 0 & 0 & 0 \\
* & * & * & * & * & \Upsilon_{66 i} & \Upsilon_{67 i} & \Upsilon_{68 i} & 0 \\
* & * & * & * & * & * & \Upsilon_{77 i} & \Upsilon_{78 i} & 0 \\
* & * & * & * & * & * & * & \Upsilon_{88 i} & 0 \\
* & * & * & * & * & * & * & * & -\psi
\end{array}\right], \\
& \Upsilon_{11 i}=\sum_{l=1}^{N} \pi_{i l} E^{T} P_{l}+P_{l} A+A^{T} P_{l}+G^{T} P_{l} G-\lambda_{1} \breve{R}_{1} \text {, } \\
& \Upsilon_{16 i}=-\lambda_{1} \breve{R}_{2}+P_{l} B+G^{T} P_{l} H, \\
& \Upsilon_{17 i}=P_{l} B_{d}+G^{T} P_{l} H_{d}, \quad \Upsilon_{18 i}=P_{l} C+G^{T} P_{l} D, \quad \Upsilon_{27 i}=-\lambda_{2} \breve{S}_{2}+G_{d}^{T} P_{l} H_{d}, \\
& \Upsilon_{33 i}=\sum_{j=1}^{N} \pi_{i j} \tilde{E}^{T} P_{j}+W^{T} U W+U_{i}+d_{1} W^{T} \tilde{U} W+P_{i} \tilde{A} \\
& +\tilde{A}^{T} P_{i}+W^{T} \tilde{G}^{T} P_{i} \tilde{G} W+d_{1} X_{11}+\bar{N}_{1 i}+\bar{N}_{1 i}^{T}-\tilde{L}^{T} Q \tilde{L}, \\
& \Upsilon_{34 i}=P_{i} \tilde{A}_{d}+W^{T} \tilde{G}^{T} P_{i} \tilde{G}_{d} W+d_{1} X_{12}-\bar{N}_{1 i}+\bar{N}_{2 i}^{T}+\bar{M}_{1 i}-\tilde{L}^{T} Q \tilde{J}, \\
& \Upsilon_{36 i}=P_{i} \tilde{B}+W^{T} \tilde{G}^{T} P_{i} \tilde{H}-\tilde{L}^{T} Q \tilde{B}_{z i}, \quad \Upsilon_{37 i}=P_{i} \tilde{B}_{d}+W^{T} \tilde{G}^{T} P_{i} \tilde{H}_{d}-\tilde{L}^{T} Q \tilde{H}_{z i}, \\
& \Upsilon_{38 i}=P_{i} \tilde{C}+W^{T} \tilde{G}^{T} P_{i} \tilde{D}-\tilde{L}^{T} Q \tilde{K}-\tilde{L}^{T} S, \\
& \Upsilon_{44 i}=-\left(1-h_{i}\right) W^{T} U W+W^{T} \tilde{G}_{d}^{T} P_{i} \tilde{G}_{d} W+d_{1} X_{22} \\
& -\bar{N}_{2 i}-\bar{N}_{2 i}^{T}+\bar{M}_{2 i}^{T}+\bar{M}_{2 i}-\tilde{J}^{T} Q \tilde{J} \text {, } \\
& \Upsilon_{46 i}=W^{T} \tilde{G}_{d}^{T} P_{i} \tilde{H}-\tilde{J}^{T} Q \tilde{B}_{z i}, \quad \Upsilon_{47 i}=W^{T} \tilde{G}_{d}^{T} P_{i} \tilde{H}_{d}-\tilde{J}^{T} Q \tilde{H}_{z i}, \\
& \Upsilon_{48 i}=W^{T} \tilde{G}_{d}^{T} P_{i} \tilde{D}-\tilde{J}^{T} Q \tilde{K}-\tilde{J}^{T} S, \quad \Upsilon_{66 i}=\tilde{H}^{T} P_{i} \tilde{H}-\lambda_{1} I+H^{T} P_{l} H-\tilde{B}_{z i}^{T} Q \tilde{B}_{z i},
\end{aligned}
$$




$$
\begin{aligned}
& \Upsilon_{67 i}=\tilde{H}^{T} P_{i} \tilde{H}_{d}+H^{T} P_{l} H_{d}-\tilde{B}_{z i}^{T} Q \tilde{H}_{z i}, \\
& \Upsilon_{68 i}=\tilde{H}^{T} P_{i} \tilde{D}-\tilde{B}_{z i}^{T} Q \tilde{K}-\tilde{B}_{z i}^{T} S+H^{T} P_{l} D, \\
& \Upsilon_{77 i}=\tilde{H}_{d}^{T} P_{i} \tilde{H}_{d}-\lambda_{2} I+H_{d}^{T} P_{l} H_{d}-\tilde{H}_{z i}^{T} Q \tilde{H}_{z i}, \\
& \Upsilon_{78 i}=\tilde{H}_{d}^{T} P_{i} \tilde{D}-\tilde{H}_{z i}^{T} Q \tilde{K}-\tilde{H}_{z i}^{T} S+H_{d}^{T} P_{l} D, \\
& \Upsilon_{88 i}=\tilde{D}^{T} P_{i} \tilde{D}-\tilde{K}^{T} Q \tilde{K}-S^{T} \tilde{K}-\tilde{K}^{T} S-R+D^{T} P_{l} D .
\end{aligned}
$$

When $\Upsilon_{i}<0$, there must exist $\alpha>0$ small enough such that $\Upsilon_{i}+\alpha \operatorname{diag}(0,0,0,0,0,0,0, I$, $0,0)<0$. Hence, there exists $\alpha>0$ for all $T>0$ and $0 \neq v(t) \in L_{2}[0, \infty)$, we have $J(T, \alpha) \geq 0$. By the Schur complement formula, (13) is completed.

Based on the sufficient conditions above, the following criterion can be obtained readily.

Theorem 2 Given matrices $Q, S, R$, where $Q, R$ are symmetric and $Q$ is negative definite and with all uncertainties, the robust dissipative filtering problem is solved for the uncertain Markovian jump system (9) if there exist positive scalars $\lambda_{1}, \lambda_{2}$ and $\varepsilon_{k i}(k=1,2)$, where $i$ is taking values in a finite set $S=\{1,2, \ldots, N\}$, symmetric matrices $P_{l}>0, X_{i}>0, Y_{i}>0, U>0$, $U_{i}>0$ and $\tilde{U}>0$, matrices $W_{i}, Z_{i}, V_{i}, R_{i}$, and any appropriately dimensioned matrices $N_{i}=\left[\bar{N}_{1 i}^{T} \bar{N}_{2 i}^{T}\right]^{T}, M_{i}=\left[\bar{M}_{1 i}^{T} \bar{M}_{2 i}^{T}\right]^{T}, X=X^{T}=\left[\begin{array}{cc}X_{11} & X_{12} \\ * & X_{22}\end{array}\right] \geq 0$ such that the following LMI holds:

$$
\begin{aligned}
& E^{T} P_{l}=P_{l} E \geq 0, \\
& E^{T} X_{i}=X_{i} E \geq 0, \\
& R_{i}^{T}=R_{i} \geq 0,
\end{aligned}
$$

$$
\left[\begin{array}{ccc}
\Theta_{1 i} & \Theta_{2 i} & \Theta_{3 i} \\
* & \Theta_{4 i} & 0 \\
* & * & \Theta_{4 i}
\end{array}\right]<0,
$$

where

$$
\Theta_{1 i}=\left[\begin{array}{cccccccccccccccc}
\Theta_{11} & \Theta_{12} & 0 & 0 & 0 & 0 & 0 & 0 & \Theta_{19} & \Theta_{110} & P_{l} C_{i} & 0 & 0 & \Theta_{114} & 0 & 0 \\
* & \Theta_{22} & 0 & 0 & 0 & 0 & 0 & 0 & 0 & \Theta_{210} & 0 & 0 & 0 & \Theta_{214} & 0 & 0 \\
* & * & \Theta_{33} & \Theta_{34} & \Theta_{35} & \Theta_{36} & \Theta_{37} & \Theta_{38} & \Theta_{39} & \Theta_{310} & \Theta_{311} & 0 & \Theta_{313} & 0 & \Theta_{315} & \Theta_{316} \\
* & * & * & \Theta_{44} & \Theta_{45} & \Theta_{46} & \Theta_{47} & \Theta_{48} & \Theta_{49} & \Theta_{410} & \Theta_{411} & 0 & \Theta_{413} & 0 & 0 & 0 \\
* & * & * & * & \Theta_{55} & \Theta_{56} & \Theta_{57} & \Theta_{58} & 0 & 0 & \Theta_{511} & 0 & \Theta_{513} & 0 & \Theta_{515} & \Theta_{516} \\
* & * & * & * & * & \Theta_{66} & \Theta_{67} & \Theta_{68} & 0 & 0 & \Theta_{611} & 0 & \Theta_{613} & 0 & 0 & 0 \\
* & * & * & * & * & * & \Theta_{77} & 0 & 0 & 0 & 0 & 0 & 0 & 0 & 0 & 0 \\
* & * & * & * & * & * & * & \Theta_{88} & 0 & 0 & 0 & 0 & 0 & 0 & 0 & 0 \\
* & * & * & * & * & * & * & * & \Theta_{99} & 0 & \Theta_{911} & 0 & \Theta_{913} & \Theta_{914} & \Theta_{915} & \Theta_{916} \\
* & * & * & * & * & * & * & * & * & \eta_{1} & \eta_{2} & 0 & \eta_{3} & \eta_{4} & \eta_{5} & \eta_{6} \\
* & * & * & * & * & * & * & * & * & * & \eta_{7} & 0 & \eta_{8} & \eta_{9} & \eta_{10} & \eta_{11} \\
* & * & * & * & * & * & * & * & * & * & * & -\psi & 0 & 0 & 0 & 0 \\
* & * & * & * & * & * & * & * & * & * & * & * & Q & 0 & 0 & 0 \\
* & * & * & * & * & * & * & * & * & * & * & * & * & \eta_{12} & 0 & 0 \\
* & * & * & * & * & * & * & * & * & * & * & * & * & * & \eta_{13} & 0 \\
* & * & * & * & * & * & * & * & * & * & * & * & * & * & * & -Y_{i}
\end{array}\right],
$$




$$
\begin{aligned}
& \Theta_{2 i}=\left[\begin{array}{ccccccc}
P_{l} M_{i 1} & 0 & 0 & 0 & 0 & 0 & 0 \\
0 & 0 & 0 & 0 & 0 & 0 & 0 \\
0 & X_{i} M_{i 1} & 0 & 0 & 0 & 0 & 0 \\
0 & Y_{i} M_{i 1}-Z_{i} M_{i 3} & -M_{\Sigma} & -Y_{i} M_{i 7} & -Y_{i} M_{i 6} & 0 & 0 \\
0 & 0 & 0 & 0 & 0 & 0 & 0 \\
0 & 0 & 0 & 0 & 0 & 0 & 0 \\
0 & 0 & 0 & 0 & 0 & 0 & 0 \\
0 & 0 & 0 & 0 & 0 & 0 & 0 \\
0 & 0 & 0 & 0 & 0 & 0 & 0 \\
0 & 0 & 0 & 0 & 0 & 0 & 0 \\
0 & -S^{T} M_{i 5} & 0 & 0 & 0 & S^{T} M_{i 7} & 0 \\
0 & 0 & 0 & 0 & 0 & 0 & 0 \\
0 & Q M_{i 5} & 0 & 0 & 0 & -Q M_{i 7} & 0 \\
P_{l} M_{i 2} & 0 & 0 & 0 & 0 & 0 & 0 \\
0 & X_{i} M_{i 2} & 0 & 0 & 0 & 0 & 0 \\
0 & Y_{i} M_{i 2}-Z_{i} M_{i 4} & 0 & 0 & 0 & 0 & Y_{i} M_{i 6}
\end{array}\right], \\
& \Theta_{3 i}=\left[\begin{array}{ccccccc}
\varepsilon_{1 i} N_{i 1}^{T} & 0 & 0 & 0 & 0 & 0 & 0 \\
\varepsilon_{1 i} N_{i 2}^{T} & 0 & 0 & 0 & 0 & 0 & 0 \\
0 & \varepsilon_{1 i} N_{i 1}^{T} & 0 & 0 & \varepsilon_{2 i} N_{i 7}^{T}+\varepsilon_{2 i} \bar{A}_{i}^{T} N_{i 8}^{T} & \varepsilon_{2 i} N_{i 7}^{T} & -\varepsilon_{2 i} \bar{G}_{i}^{T} N_{i 8}^{T} \\
0 & 0 & \varepsilon_{2 i} N_{\Sigma}^{T} & 0 & -\varepsilon_{2 i} N_{i 7}^{T} & -\varepsilon_{2 i} N_{i 7}^{T} & 0 \\
0 & \varepsilon_{1 i} N_{i 2}^{T} & 0 & \varepsilon_{2 i} N_{i 6}^{T} & \varepsilon_{2 i} \bar{A}_{d i}^{T} N_{i 8}^{T} & \varepsilon_{2 i} N_{i 8}^{T} & -\varepsilon_{2 i} \bar{G}_{d i}^{T} N_{i 8}^{T} \\
0 & 0 & 0 & -\varepsilon_{2 i} N_{i 6}^{T} & 0 & -\varepsilon_{2 i} N_{i 8}^{T} & 0 \\
0 & 0 & 0 & 0 & 0 & 0 & 0 \\
0 & 0 & 0 & 0 & 0 & 0 & 0 \\
\varepsilon_{1 i} N_{i 3}^{T} & \varepsilon_{1 i} N_{i 3}^{T} & 0 & 0 & \varepsilon_{2 i} \bar{B}_{i}^{T} N_{i 8}^{T} & 0 & -\varepsilon_{2 i} \bar{H}_{i}^{T} N_{i 8}^{T} \\
\varepsilon_{1 i} N_{i 4}^{T} & \varepsilon_{1 i} N_{i 4}^{T} & 0 & 0 & \varepsilon_{2 i} \bar{B}_{-i d}^{T} N_{i 8}^{T} & 0 & -\varepsilon_{2 i} \bar{H}_{d i}^{T} N_{i 8}^{T} \\
\varepsilon_{1 i} N_{i 5}^{T} & \varepsilon_{1 i} N_{i 5}^{T} & 0 & 0 & \varepsilon_{2 i} \bar{C}_{i}^{T} N_{i 8}^{T} & 0 & -\varepsilon_{2 i} \bar{D}_{i}^{T} N_{i 8}^{T} \\
0 & 0 & 0 & 0 & 0 & 0 & 0 \\
0 & 0 & 0 & 0 & 0 & 0 & 0 \\
0 & 0 & 0 & 0 & 0 & 0 & 0 \\
0 & 0 & 0 & 0 & 0 & 0 & 0 \\
0 & 0 & 0 & 0 & 0 & 0 & 0
\end{array}\right], \\
& \Theta_{4 i}=\operatorname{diag}\left\{-\varepsilon_{1 i} I,-\varepsilon_{1 i} I,-\varepsilon_{2 i} I,-\varepsilon_{2 i} I,-\varepsilon_{2 i} I,-\varepsilon_{2 i} I,-\varepsilon_{2 i} I\right\}, \\
& \Theta_{11}=\sum_{l=1}^{N} \pi_{i l} E^{T} P_{l}+P_{l} A_{i}+A_{i}^{T} P_{l}-\lambda_{1} \breve{R}_{1}, \quad \Theta_{12}=P_{l} A_{d i}, \quad \Theta_{19}=-\lambda_{1} \breve{R}_{2}+P_{l} B_{i}, \\
& \Theta_{110}=P_{l} B_{d i}, \quad \Theta_{114}=G_{i}^{T} P_{l}, \quad \Theta_{22}=-\lambda_{2} \breve{S}_{1}, \\
& \Theta_{210}=-\lambda_{2} \breve{S}_{2}, \quad \Theta_{214}=G_{d i}^{T} P_{l}, \\
& \Theta_{33}=\sum_{j=1}^{N} \pi_{i j} E^{T} X_{j}+U+U_{i 1}+d_{1} \tilde{U}+X_{i} A_{i}+A_{i}^{T} X_{i}+d_{1} X_{1111}+N_{1 i 11}+N_{1 i 11}^{T}, \\
& \Theta_{34}=A_{i}^{T} Y_{i}-W_{i}^{T}-\bar{A}_{i}^{T} Z_{i}^{T}+d_{1} X_{1112}+N_{1 i 12}+N_{1 i 21}^{T} \text {, } \\
& \Theta_{35}=X_{i} A_{d i}+d_{1} X_{1211}-N_{1 i 11}+N_{2 i 11}^{T}+M_{1 i 11}, \quad \Theta_{36}=d_{1} X_{1212}-N_{1 i 12}+N_{2 i 21}^{T}+M_{1 i 12} \text {, } \\
& \Theta_{37}=-M_{1 i 11}, \quad \Theta_{38}=-M_{1 i 12}, \quad \Theta_{39}=X_{i} B_{i}, \\
& \Theta_{310}=X_{i} B_{d i}, \quad \Theta_{311}=X_{i} C_{i}-L_{i}^{T} S+L_{f i}^{T} S, \\
& \Theta_{313}=L_{i}^{T} Q-L_{f i}^{T} Q, \quad \Theta_{315}=G_{i}^{T} X_{i}, \quad \Theta_{316}=G_{i}^{T} Y_{i}-\bar{G}_{i}^{T} Z_{i}^{T},
\end{aligned}
$$




$$
\begin{aligned}
& \Theta_{44}=\sum_{j=1}^{N} \pi_{i j} R_{j}+W_{i}+W_{i}^{T}+U_{i 2}+d_{1} X_{1122}+N_{1 i 22}+N_{1 i 22}^{T} \\
& \Theta_{45}=Y_{i} A_{d i}-V_{i}-Z_{i} \bar{A}_{d i}+d_{1} X_{1221}-N_{1 i 21}+N_{2 i 12}^{T}+M_{1 i 21}, \\
& \Theta_{46}=V_{i}+d_{1} X_{1222}-N_{1 i 22}+N_{2 i 22}^{T}+M_{1 i 22}, \quad \Theta_{47}=-M_{1 i 21}, \quad \Theta_{48}=-M_{1 i 22}, \\
& \Theta_{49}=Y_{i} B_{i}-Z_{i} \bar{B}_{i}, \quad \Theta_{410}=Y_{i} B_{d i}-Z_{i} \bar{B}_{d i}, \\
& \Theta_{411}=Y_{i} C_{i}-Z_{i} \bar{C}_{i}-L_{f i}^{T} S, \quad \Theta_{413}=L_{f i}^{T} Q, \\
& \Theta_{55}=-\left(1-h_{i}\right) U+d_{1} X_{2211}-N_{2 i 11}-N_{2 i 11}^{T}+M_{2 i 11}^{T}+M_{2 i 11}, \\
& \Theta_{56}=d_{1} X_{2212}-N_{2 i 12}-N_{2 i 21}^{T}+M_{2 i 21}^{T}+M_{2 i 12}, \quad \Theta_{57}=-M_{2 i 11}, \quad \Theta_{58}=-M_{2 i 12} \text {, } \\
& \Theta_{511}=-J_{i}^{T} S+J_{f i}^{T} S, \quad \Theta_{513}=J_{i}^{T} Q-J_{f i}^{T} Q, \\
& \Theta_{515}=G_{d i}^{T} X_{i}, \quad \Theta_{516}=G_{d i}^{T} Y_{i}-\bar{G}_{d i}^{T} Z_{i}^{T}, \\
& \Theta_{66}=d_{1} X_{2222}-N_{2 i 22}-N_{2 i 22}^{T}+M_{2 i 22}^{T}+M_{2 i 22}, \quad \Theta_{67}=-M_{2 i 21}, \quad \Theta_{68}=-M_{2 i 22} \text {, } \\
& \Theta_{611}=-J_{f i}^{T} S, \quad \Theta_{613}=J_{f i}^{T} Q, \quad \Theta_{77}=-U_{i 1}, \\
& \Theta_{88}=-U_{i 2}, \quad \Theta_{99}=-\lambda_{1} I, \quad \Theta_{911}=-B_{z i}^{T} S, \\
& \Theta_{913}=B_{z i}^{T} Q, \quad \Theta_{914}=H_{i}^{T} P_{l}, \quad \Theta_{915}=H_{i}^{T} X_{i}, \\
& \Theta_{916}=H_{i}^{T} Y_{i}-\bar{H}_{i}^{T} Z_{i}^{T}, \quad \eta_{1}=-\lambda_{2} I, \quad \eta_{2}=-H_{z i}^{T} S, \\
& \eta_{3}=H_{z i}^{T} Q, \quad \eta_{4}=H_{d i}^{T} P_{l}, \quad \eta_{5}=H_{d i}^{T} X_{i}, \\
& \eta_{6}=H_{d i}^{T} Y_{i}-\bar{H}_{d i}^{T} Z_{i}^{T}, \quad \eta_{7}=-K_{i}^{T} S-S^{T} K_{i}-R, \\
& \eta_{8}=K_{i}^{T} Q, \quad \eta_{9}=D_{i}^{T} P_{l}, \quad \eta_{10}=D_{i}^{T} X_{i}, \\
& \eta_{11}=D_{i}^{T} Y_{i}-\bar{D}_{i}^{T} Z_{i}^{T}, \quad \eta_{12}=-P_{l}, \quad \eta_{13}=-X_{i}, \\
& M_{\Sigma}=\left[\begin{array}{llll}
\pi_{i 1} Y_{1} M_{16} & \pi_{i 2} Y_{2} M_{26} & \cdots & \pi_{i, i-1} Y_{i-1} M_{i-1,6}
\end{array}\right. \\
& \left.\pi_{i, i+1} Y_{i+1} M_{i+1,6} \quad \cdots \quad \pi_{i, N} Y_{N} M_{N, 6}\right], \\
& N_{\Sigma}=\left[\begin{array}{llllll}
N_{16}^{T} N_{26}^{T} & \cdots & N_{i-1,6}^{T} & N_{i+1,6}^{T} & \cdots & N_{N, 6}^{T}
\end{array}\right]^{T}, \\
& \psi=\left[\begin{array}{ll}
\psi_{1} & \\
& \psi_{2}
\end{array}\right], \quad \psi_{1}=\left[\begin{array}{ccccc}
X_{11} & X_{12} & 0 & 0 & \bar{N}_{1 i} \\
* & X_{22} & 0 & 0 & \bar{N}_{2 i} \\
* & * & \tilde{U} & 0 & 0 \\
* & * & * & 0 & 0 \\
* & * & * & * & 0
\end{array}\right], \\
& \psi_{2}=\left[\begin{array}{ccccc}
X_{11} & X_{12} & 0 & 0 & \bar{M}_{1 i} \\
* & X_{22} & 0 & 0 & \bar{M}_{2 i} \\
* & * & \tilde{U} & 0 & 0 \\
* & * & * & 0 & 0 \\
* & * & * & * & 0
\end{array}\right], \\
& U_{i}=\left[\begin{array}{ll}
U_{i 1} & \\
& U_{i 2}
\end{array}\right], \quad X_{11}=\left[\begin{array}{ll}
X_{1111} & X_{1112} \\
X_{1121} & X_{1122}
\end{array}\right] \text {, }
\end{aligned}
$$




$$
\begin{array}{ll}
X_{12}=\left[\begin{array}{ll}
X_{1211} & X_{1212} \\
X_{1221} & X_{1222}
\end{array}\right], & X_{22}=\left[\begin{array}{ll}
X_{2211} & X_{2212} \\
X_{2221} & X_{2222}
\end{array}\right], \\
\bar{N}_{1 i}=\left[\begin{array}{ll}
N_{1 i 11} & N_{1 i 12} \\
N_{1 i 21} & N_{1 i 22}
\end{array}\right], & \bar{N}_{2 i}=\left[\begin{array}{ll}
N_{2 i 11} & N_{2 i 12} \\
N_{2 i 21} & N_{2 i 22}
\end{array}\right], \\
\bar{M}_{1 i}=\left[\begin{array}{ll}
M_{1 i 11} & M_{1 i 12} \\
M_{1 i 21} & M_{1 i 22}
\end{array}\right], & \bar{M}_{2 i}=\left[\begin{array}{ll}
M_{2 i 11} & M_{2 i 12} \\
M_{2 i 21} & M_{2 i 22}
\end{array}\right] .
\end{array}
$$

In this case, a suitable robust dissipative filter in the form of (6) has parameters as follows:

$$
A_{f i}=Y_{i}^{-1} W_{i}, \quad B_{f i}=Y_{i}^{-1} Z_{i}, \quad C_{f i}=Y_{i}^{-1} V_{i}, \quad L_{f i}, J_{f}, E_{f i}=Y_{i}^{-1} R_{i} .
$$

Remark 3 The robust dissipative filter design problem is solved in Theorem 2 for the uncertain stochastic singular time-delay systems with Markovian jump parameters. We derive an LMI-based sufficient condition for the existence of full-order filters that ensure asymptotic stability and strict robust $(Q, S, R)$ dissipativity of the resulting filtering error system and reduce the effect of the disturbance input on the estimated signal to a prescribed level for all admissible uncertainties. The feasibility of the filter design problem can be readily checked by the solvability of an LMI, which is dependent on the upper bound of the time-varying delays. The solvability of such a delay-dependent LMI can be readily checked by resorting to the Matlab LMI Tool box. In the next section, an illustrative example is provided to show the potential of the proposed techniques.

Remark 4 When the finite set $S=\{1\}$, the uncertain linear stochastic system (1) is a generalized uncertain stochastic singular time-delay system without Markovian jump parameters.

\section{Numerical value example}

Example In this section, a numerical example is presented to demonstrate the usefulness of the developed method on the design of a robust dissipative filter for the uncertain stochastic singular time-delay systems with Markovian jump parameters.

Consider system (1) with the following parameters:

$$
\begin{aligned}
& A_{1}=\left[\begin{array}{ll}
1 & 0 \\
1 & 0
\end{array}\right], \quad A_{d 1}=\left[\begin{array}{ll}
2 & 1 \\
1 & 2
\end{array}\right], \quad B_{1}=\left[\begin{array}{ll}
3 & 2 \\
4 & 1
\end{array}\right], \\
& B_{d 1}=\left[\begin{array}{ll}
3 & 2 \\
4 & 1
\end{array}\right], \quad C_{1}=\left[\begin{array}{ll}
1 & 3 \\
1 & 2
\end{array}\right] \text {, } \\
& G_{1}=\left[\begin{array}{ll}
2 & 4 \\
1 & 2
\end{array}\right], \quad G_{d 1}=\left[\begin{array}{ll}
1 & 2 \\
1 & 1
\end{array}\right], \quad H_{1}=\left[\begin{array}{ll}
2 & 1 \\
1 & 2
\end{array}\right] \text {, } \\
& H_{d 1}=\left[\begin{array}{ll}
2 & 1 \\
1 & 2
\end{array}\right], \quad D_{1}=\left[\begin{array}{ll}
2 & 1 \\
1 & 2
\end{array}\right] \text {, } \\
& \bar{A}_{1}=\left[\begin{array}{ll}
1 & 0 \\
1 & 0
\end{array}\right], \quad \bar{A}_{d 1}=\left[\begin{array}{ll}
2 & 1 \\
1 & 2
\end{array}\right], \quad \bar{B}_{1}=\left[\begin{array}{ll}
3 & 2 \\
4 & 1
\end{array}\right],
\end{aligned}
$$




$$
\begin{aligned}
& \bar{B}_{d 1}=\left[\begin{array}{ll}
3 & 2 \\
4 & 1
\end{array}\right], \quad \bar{C}_{1}=\left[\begin{array}{ll}
1 & 3 \\
1 & 2
\end{array}\right], \\
& \bar{G}_{1}=\left[\begin{array}{ll}
2 & 4 \\
1 & 2
\end{array}\right], \quad \bar{G}_{d 1}=\left[\begin{array}{ll}
1 & 2 \\
1 & 1
\end{array}\right], \quad \bar{H}_{1}=\left[\begin{array}{ll}
2 & 1 \\
1 & 2
\end{array}\right], \\
& \bar{H}_{d 1}=\left[\begin{array}{ll}
2 & 1 \\
1 & 2
\end{array}\right], \quad \bar{D}_{1}=\left[\begin{array}{ll}
2 & 1 \\
1 & 2
\end{array}\right] \\
& L_{1}=\left[\begin{array}{ll}
1 & 0 \\
1 & 0
\end{array}\right], \quad J_{1}=\left[\begin{array}{ll}
2 & 1 \\
1 & 2
\end{array}\right], \quad B_{z 1}=\left[\begin{array}{ll}
3 & 2 \\
4 & 1
\end{array}\right] \text {, } \\
& H_{z 1}=\left[\begin{array}{ll}
3 & 2 \\
4 & 1
\end{array}\right], \quad K_{1}=\left[\begin{array}{ll}
1 & 3 \\
3 & 1
\end{array}\right] \text {, } \\
& M_{11}=\left[\begin{array}{l}
0.01 \\
0.02
\end{array}\right], \quad M_{12}=\left[\begin{array}{c}
-0.02 \\
0.02
\end{array}\right], \quad M_{13}=\left[\begin{array}{c}
-0.02 \\
0.01
\end{array}\right] \text {, } \\
& M_{14}=\left[\begin{array}{l}
0.01 \\
0.02
\end{array}\right], \quad M_{15}=\left[\begin{array}{c}
-0.02 \\
0.02
\end{array}\right] \text {, } \\
& N_{11}=\left[\begin{array}{ll}
0.01 & 0.02
\end{array}\right], \quad N_{12}=\left[\begin{array}{ll}
-0.02 & 0.02
\end{array}\right], \quad N_{13}=\left[\begin{array}{ll}
-0.02 & 0.01
\end{array}\right], \\
& N_{14}=\left[\begin{array}{ll}
0.01 & 0.02
\end{array}\right], \quad N_{15}=\left[\begin{array}{ll}
-0.02 & 0.02
\end{array}\right] \text {, } \\
& A_{2}=\left[\begin{array}{ll}
1 & 0 \\
1 & 0
\end{array}\right], \quad A_{d 2}=\left[\begin{array}{ll}
2 & 1 \\
1 & 2
\end{array}\right], \quad B_{2}=\left[\begin{array}{ll}
3 & 2 \\
4 & 1
\end{array}\right], \\
& B_{d 2}=\left[\begin{array}{ll}
3 & 2 \\
4 & 1
\end{array}\right], \quad C_{2}=\left[\begin{array}{ll}
1 & 3 \\
1 & 2
\end{array}\right] \text {, } \\
& G_{2}=\left[\begin{array}{ll}
2 & 4 \\
1 & 2
\end{array}\right], \quad G_{d 2}=\left[\begin{array}{ll}
1 & 2 \\
1 & 1
\end{array}\right], \quad H_{2}=\left[\begin{array}{ll}
2 & 1 \\
1 & 2
\end{array}\right], \\
& H_{d 2}=\left[\begin{array}{ll}
2 & 1 \\
1 & 2
\end{array}\right], \quad D_{2}=\left[\begin{array}{ll}
2 & 1 \\
1 & 2
\end{array}\right] \text {, } \\
& \bar{A}_{2}=\left[\begin{array}{ll}
1 & 0 \\
1 & 0
\end{array}\right], \quad \bar{A}_{d 2}=\left[\begin{array}{ll}
2 & 1 \\
1 & 2
\end{array}\right], \quad \bar{B}_{2}=\left[\begin{array}{ll}
3 & 2 \\
4 & 1
\end{array}\right], \\
& \bar{B}_{d 2}=\left[\begin{array}{ll}
3 & 2 \\
4 & 1
\end{array}\right], \quad \bar{C}_{2}=\left[\begin{array}{ll}
1 & 3 \\
1 & 2
\end{array}\right], \\
& \bar{G}_{2}=\left[\begin{array}{ll}
2 & 4 \\
1 & 2
\end{array}\right], \quad \bar{G}_{d 2}=\left[\begin{array}{ll}
1 & 2 \\
1 & 1
\end{array}\right], \quad \bar{H}_{2}=\left[\begin{array}{ll}
2 & 1 \\
1 & 2
\end{array}\right] \text {, } \\
& \bar{H}_{d 2}=\left[\begin{array}{ll}
2 & 1 \\
1 & 2
\end{array}\right], \quad \bar{D}_{2}=\left[\begin{array}{ll}
2 & 1 \\
1 & 2
\end{array}\right] \text {, } \\
& L_{2}=\left[\begin{array}{ll}
1 & 0 \\
1 & 0
\end{array}\right], \quad J_{2}=\left[\begin{array}{ll}
2 & 1 \\
1 & 2
\end{array}\right], \quad B_{z 2}=\left[\begin{array}{ll}
3 & 2 \\
4 & 1
\end{array}\right] \text {, }
\end{aligned}
$$




$$
\begin{aligned}
& H_{z 2}=\left[\begin{array}{ll}
3 & 2 \\
4 & 1
\end{array}\right], \quad K_{2}=\left[\begin{array}{ll}
1 & 3 \\
3 & 1
\end{array}\right], \\
& M_{21}=\left[\begin{array}{l}
0.01 \\
0.02
\end{array}\right], \quad M_{22}=\left[\begin{array}{c}
-0.02 \\
0.02
\end{array}\right], \quad M_{23}=\left[\begin{array}{c}
-0.02 \\
0.01
\end{array}\right], \\
& M_{24}=\left[\begin{array}{l}
0.01 \\
0.02
\end{array}\right], \quad M_{25}=\left[\begin{array}{c}
-0.02 \\
0.02
\end{array}\right], \\
& N_{21}=\left[\begin{array}{ll}
0.01 & 0.02
\end{array}\right], \quad N_{22}=\left[\begin{array}{ll}
-0.02 & 0.02
\end{array}\right], \quad N_{23}=\left[\begin{array}{ll}
-0.02 & 0.01
\end{array}\right], \\
& N_{24}=\left[\begin{array}{ll}
0.01 & 0.02
\end{array}\right], \quad N_{25}=\left[\begin{array}{ll}
-0.02 & 0.02
\end{array}\right], \quad \\
& \hat{R}_{1}=\left[\begin{array}{ll}
1 & 0 \\
1 & 0
\end{array}\right], \quad \hat{R}_{2}=\left[\begin{array}{ll}
2 & 1 \\
1 & 2
\end{array}\right], \quad \hat{S}_{1}=\left[\begin{array}{ll}
3 & 2 \\
4 & 1
\end{array}\right], \quad \hat{S}_{2}=\left[\begin{array}{ll}
3 & 2 \\
4 & 1
\end{array}\right],
\end{aligned}
$$

and the time-varying delay $d_{i}(t)$ satisfies (2) with $d_{1}=1, h_{1}=0.2, h_{2}=0.5$. The transition probability matrix is supposed to be

$$
M=\left[\begin{array}{cc}
-0.5 & 0.5 \\
0.3 & -0.3
\end{array}\right] \text {. }
$$

The filter uncertainties are in the form of (8) with

$$
\begin{aligned}
& M_{16}=\left[\begin{array}{l}
0.01 \\
0.02
\end{array}\right], \quad M_{17}=\left[\begin{array}{c}
-0.02 \\
0.02
\end{array}\right], \quad N_{16}=\left[\begin{array}{ll}
0.01 & 0.02
\end{array}\right], \\
& N_{17}=\left[\begin{array}{ll}
-0.02 & 0.02
\end{array}\right], \quad N_{18}=\left[\begin{array}{ll}
-0.02 & 0.01
\end{array}\right], \\
& M_{26}=\left[\begin{array}{l}
0.01 \\
0.02
\end{array}\right], \quad M_{27}=\left[\begin{array}{c}
-0.02 \\
0.02
\end{array}\right], \quad N_{26}=\left[\begin{array}{ll}
0.01 & 0.02
\end{array}\right], \\
& N_{27}=\left[\begin{array}{ll}
-0.02 & 0.02
\end{array}\right], \quad N_{28}=\left[\begin{array}{ll}
-0.02 & 0.01
\end{array}\right] .
\end{aligned}
$$

Suppose $Q=\operatorname{diag}(-1,-1), R=\operatorname{diag}(1,1), S=\operatorname{diag}(1,1)$. With the above parameters and by using the Matlab LMI Tool box, we solve LMI (20) and obtain

$$
\begin{array}{ll}
A_{f 1}=\left[\begin{array}{ll}
-0.4506 & -0.1244 \\
-0.1277 & -0.5264
\end{array}\right], & B_{f 1}=\left[\begin{array}{cc}
1.0000 & -0.0000 \\
-0.0000 & 1.0000
\end{array}\right], \\
C_{f 1}=\left[\begin{array}{ll}
-0.0818 & -0.0242 \\
-0.0236 & -0.0946
\end{array}\right], & \\
L_{f 1}=\left[\begin{array}{cc}
1.9268 & -0.4997 \\
-0.2300 & 0.0943
\end{array}\right], & J_{f 1}=\left[\begin{array}{cc}
1.0139 & 0.4922 \\
0.4915 & 1.0000
\end{array}\right], \\
E_{f 1}=\left[\begin{array}{ll}
1.4887 & -0.0282 \\
-0.1388 & 1.4305
\end{array}\right], & \\
A_{f 2}=\left[\begin{array}{ll}
-0.4564 & -0.1298 \\
-0.1310 & -0.5322
\end{array}\right], & B_{f 2}=\left[\begin{array}{cc}
1.0000 & -0.0000 \\
-0.0000 & 1.0000
\end{array}\right],
\end{array}
$$




$$
\begin{array}{rlr}
C_{f 2} & =\left[\begin{array}{ll}
-0.0825 & -0.0245 \\
-0.0241 & -0.0950
\end{array}\right], \\
L_{f 2} & =\left[\begin{array}{cc}
1.9246 & -0.5001 \\
-0.2324 & 0.0931
\end{array}\right], \quad J_{f 2}=\left[\begin{array}{cc}
1.0153 & 0.4942 \\
0.4950 & 1.0009
\end{array}\right], \\
E_{f 2} & =\left[\begin{array}{cc}
2.0232 & -0.0278 \\
-0.1384 & 1.9660
\end{array}\right] .
\end{array}
$$

In order to show the advantage of the proposed method, we consider the filtering with $\Delta A_{f i}=0, \Delta B_{f i}=0, \Delta C_{f i}=0, \Delta L_{f i}=0, \Delta J_{f i}=0, \Delta E_{f i}=0$. And all the other parameters are the same as those above. We can obtain the filter parameters as follows:

$$
\begin{array}{ll}
A_{f 1}=\left[\begin{array}{ll}
-0.4490 & -0.1154 \\
-0.1189 & -0.5210
\end{array}\right], & B_{f 1}=\left[\begin{array}{cc}
1.0000 & -0.0000 \\
-0.0000 & 1.0000
\end{array}\right], \\
C_{f 1}=\left[\begin{array}{ll}
-0.0833 & -0.0230 \\
-0.0223 & -0.0952
\end{array}\right], & \\
L_{f 1}=\left[\begin{array}{cc}
1.9240 & -0.4949 \\
-0.2145 & 0.0921
\end{array}\right], & J_{f 1}=\left[\begin{array}{cc}
1.0133 & 0.4907 \\
0.4904 & 0.9980
\end{array}\right], \\
E_{f 1}=\left[\begin{array}{cc}
1.4740 & -0.0306 \\
-0.1412 & 1.4153
\end{array}\right], & \\
A_{f 2}=\left[\begin{array}{cc}
-0.4557 & -0.1216 \\
-0.1227 & -0.5275
\end{array}\right], & B_{f 2}=\left[\begin{array}{cc}
1.0000 & -0.0000 \\
-0.0000 & 1.0000
\end{array}\right], \\
C_{f 2}=\left[\begin{array}{cc}
-0.0840 & -0.0233 \\
-0.0228 & -0.0957
\end{array}\right], & \\
L_{f 2}=\left[\begin{array}{cc}
1.9228 & -0.4955 \\
-0.2194 & 0.0912
\end{array}\right], & J_{f 2}=\left[\begin{array}{cc}
1.0147 & 0.4934 \\
0.4937 & 1.0000
\end{array}\right], \\
E_{f 2}=\left[\begin{array}{cc}
1.4743 & -0.0301 \\
-0.1408 & 1.4158
\end{array}\right] . &
\end{array}
$$

When $\Delta A_{f i}=0, \Delta B_{f i}=0, \Delta C_{f i}=0, \Delta L_{f i}=0, \Delta J_{f i}=0, \Delta E_{f i}=0$, by Theorem 2, we can obtain $d_{1 \max }$ is $1.0192 \times 10^{8}$. But in this paper, when $\Delta A_{f i} \neq 0, \Delta B_{f i} \neq 0, \Delta C_{f i} \neq 0, \Delta L_{f i} \neq$ $0, \Delta J_{f i} \neq 0, \Delta E_{f i} \neq 0$, we can obtain that $\hat{d}_{1 \max }$ is $1.3732 \times 10^{8}$. It is easy to get $d_{1}<\hat{d}_{1}$. Therefore, we can see the advantage of the proposed method.

\section{Conclusion}

The robust dissipative filtering problem of the stochastic singular systems with Markovian jump parameters influenced by both unknown deterministic and time-varying disturbance inputs and noise is treated in this paper. A numerical example is given to illustrate the usefulness and effectiveness of the proposed design method. The results of this paper can be utilized in several ways, as stand-alone fault-tolerant state estimation techniques, as an essential part of fault-tolerant state feedback controller design, as fault-sensitive filters or as a bank of filters for multiple model fault detection and isolation schemes. 


\section{Competing interests}

The authors declare that they have no competing interests.

\section{Authors' contributions}

HY carried out the main part of this manuscript. YM participated in the discussion and corrected the main theorem. All authors read and approved the final manuscript.

\section{Acknowledgements}

This paper is supported by the National Natural Science Foundation of China (No. 61273004). The authors also gratefully acknowledge the helpful comments and suggestions of the reviewers, which have improved the presentation.

\section{Received: 15 March 2013 Accepted: 24 April 2013 Published: 10 May 2013}

\section{References}

1. Boukas, EK, Liu, ZK: Deterministic and Stochastic Time Delay Systems. Birkhauser, Boston (2002)

2. Zhang, L, Boukas, EK: $H_{\infty}$ control for discrete-time Markovian jump linear systems with partly unknown transition probabilities. Int. J. Robust Nonlinear Control 19(8), 868-883 (2009)

3. Boukas, EK, Yang, H: Exponential stabilizability of stochastic systems with Markovian jumping parameters. Automatica 35(8), 1437-1441 (1999)

4. Boukas, EK: Stochastic Hybrid Systems: Analysis and Design. Birkhauser, Boston (2005)

5. Wang, GL, Zhang, QL, Sreeram, V: Partially mode-dependent $H_{\infty}$ filtering for discrete-time Markovian jump systems with partly unknown transition probabilities. Signal Process. 90(2), 548-556 (2010)

6. Wang, ZD, Liu, YR, Liu, XH: $H_{\infty}$ filtering for uncertain stochastic time-delay systems with sector-bounded nonlinearities. Automatica 5(44), 1268-1277 (2008)

7. Basin, M, Shi, P, Calderon-Alvarez, D: Joint state filtering and parameter estimation for linear stochastic time-delay systems. Signal Process. 4(91), 782-792 (2011)

8. Liu, YR, Wang, ZD, Liu, XH: Robust $H_{\infty}$ filtering for discrete nonlinear stochastic systems with time-varying delay J. Math. Anal. Appl. 1(341), 318-336 (2008)

9. Song, B, Xu, SY, Zou, Y: Non-fragile $H_{\infty}$ filtering for uncertain stochastic time-delay systems. Int. J. Innov. Comput. Inf. Control 5(8), 2257-2265 (2009)

10. Shao, HY: Delay-range-dependent robust $H_{\infty}$ filtering for uncertain stochastic systems with mode-dependent time delays and Markovian jump parameters. J. Math. Anal. Appl. 2(342), 1084-1095 (2008)

11. Chen, M, Wang, DB, Wang, ZS: Non-fragile $H_{\infty}$ filtering for uncertain stochastic time-delay systems with Markovian jump parameters. J. Syst. Eng. Electron. 32(5), 1019-1023 (2010) (in Chinese)

12. Yan, $\mathrm{HC}$, Meng, MQ-H, Zhang, $\mathrm{H}$, Shi, $\mathrm{H}$ : Robust $H_{\infty}$ exponential filtering for uncertain stochastic time-delay systems with Markovian switching and nonlinearities. Appl. Math. Comput. 215(12), 4358-4369 (2010)

13. $\mathrm{Wu}, \mathrm{ZG}, \mathrm{Su}, \mathrm{HY}, \mathrm{Chu}, \mathrm{J}$ : Robust exponential stability of uncertain singular Markovian jump time-delay systems. Acta Autom. Sin. 36(4), 558-563 (2010)

14. $\mathrm{Wu}, \mathrm{ZG}, \mathrm{Su}, \mathrm{HY}, \mathrm{Chu}, \mathrm{J}$ : Delay-dependent $H_{\infty}$ filtering for singular Markovian jump time-delay systems. Signal Process 90(6), 1815-1824 (2010)

15. Zhu, BY, Zhang, QL, Tong, SC: The passivity control for a kind of T-S fuzzy descriptor system. Syst. Eng. Theory Pract. 6(6), 81-87 (2006) (in Chinese)

16. Lo, JC, Wu, DL: Dissipative filtering for discrete fuzzy systems. In: IEEE International Conference on Fuzzy Systems, pp. 361-365. IEEE, Hong Kong (2008)

17. Li, CJ, An, HY, Feng, YF: Dissipative filtering for linear discrete-time systems via LMI. In: IEEE Chinese Control and Decision Conference (CCDC 2009), pp. 3866-3870 (2009)

18. Duan, GR, Lv, LL, Wu, AG: Robust dissipative filtering for continuous-time polytopic uncertain neutral systems. J. Syst. Eng. Electron. 20(3), 598-606 (2009)

19. Khalil, HK: Nonlinear Systems. Prentice Hall, Upper Saddle River (1996)

20. Kim, SJ, Ha, IJ: A state-space approach to analysis of almost periodic nonlinear systems with sector nonlinearities. IEEE Trans. Autom. Control 44(1), 66-70 (1999)

21. Lam, J, Gao, $H, X u, S$, Wang, $C: H_{\infty}$ and $L_{2} / H_{\infty}$ infinity model reduction for system input with sector nonlinearities. J. Optim. Theory Appl. 125(1), 137-155 (2005)

doi:10.1186/1687-1847-2013-135

Cite this article as: Ma and Yan: Delay-dependent non-fragile robust dissipative filtering for uncertain nonlinear stochastic singular time-delay systems with Markovian jump parameters. Advances in Difference Equations 2013 2013:135. 